\title{
Sur le spectre des opérateurs aux différences finies aléatoires
}

\author{
Hervé Kunz ${ }^{1}$ et Bernard Souillard ${ }^{2}$ \\ 1 Laboratoire de Physique Théorique ${ }^{\star \star}$, E.P.F.L., CH-1000 Lausanne, Suisse \\ 2 Centre de Physique Théorique ${ }^{\star}$, Ecole Polytechnique, F-91128 Palaiseau Cedex, France
}

\begin{abstract}
We study a class of random finite difference operators, a typical example of which is the finite difference Schrödinger operator with a random potential which arises in solid state physics in the tight binding approximation. We obtain with probability one, in various situations, the exact location of the spectrum, and criterions for a given part in the spectrum to be pure point or purely continuous, or for the static electric conductivity to vanish. A general formalism is developped which transforms the study of these random operators into that of the asymptotics of a multiple integral constructed from a given recipe. Finally we apply our criterions and formalism to prove that, with probability one, the one-dimensional finite difference Schrödinger operator with a random potential has pure point spectrum and developps no static conductivity.
\end{abstract}

\section{Plan}

I. Introduction - Quelques motivations physiques - Historique du sujet . . . . . . . . 201

A. Le cas général . . . . . . . . . . . . . . . . . . . . . . . . . . . . . . . 204

II. Définitions et préliminaires . . . . . . . . . . . . . . . . . . . . . . . . . . . 204

III. Détermination du spectre . . . . . . . . . . . . . . . . . . . . . . . . . . . 208

IV. Critères spectraux . . . . . . . . . . . . . . . . . . . . . . . . . . . . . . . 210

V. Sur la conductivité et la constante de diffusion . . . . . . . . . . . . . . . . . . . 217

VI. Un formalisme général . . . . . . . . . . . . . . . . . . . . . . . . . . . . 224

B. L'opérateur de Schrödinger unidimensionnel . . . . . . . . . . . . . . . . . . . 231

VII. Fonction spectrale moyenne et fonction de corrélation à volume fini . . . . . . . . . 231

VIII. Etude de l'opérateur de transfer . . . . . . . . . . . . . . . . . . . . . . . . . 235

IX. Résultats sur l'opérateur de Schrödinger unidimensionnel . . . . . . . . . . . . . 241

C. La transition de localisation vue comme un changement de phase: description qualitative 244

\section{Introduction}

Nous décrivons ici des résultats sur la nature du spectre d'une classe d'opérateurs aux différences finies aléatoires, dont un cas particulier important est celui de

* Equipe de Recherche du C.N.R.S. ${ }^{\circ} 174$

$\star \star$ Recherche soutenue par le Fonds National Suisse de la recherche scientifique 
l'opérateur de Schrödinger discrétisé avec un potentiel aléatoire $H(V)$ :

$$
H(V)=-\Delta+V
$$

agissant sur des suites de carré sommable, par exemple $\ell^{2}\left(\mathbb{Z}^{v}\right)$, par

$$
(H(V) \psi)(x)=\sum_{\substack{y \in \mathbb{Z}^{v} \\|y \in x|^{v}=1}} \psi(y)-2 v \psi(x)+V(x) \psi(x)
$$

pout tout $x \in \mathbb{Z}^{v}, \psi \in \ell^{2}\left(\mathbb{Z}^{v}\right)$. Le potentiel $V=\{V(x)\}_{x \in \mathbb{Z}^{v}}$ étant constitué par un ensemble de variables aléatoires.

Ce modèle, dans le cas où les $V(x)$ sont des variables aléatoires indépendantes identiquement distribuées, est celui qui fut utilisé par Anderson et de nombreux autres auteurs depuis pour discuter les propriétés de transport et de localisation dans divers systèmes désordonnés ${ }^{1}$. Il existe deux réalisations physiques classiques d'un tel modèle:

- les oscillateurs harmoniques avec des interactions à plus proches voisins et des masses aléatoires;

- des électrons diffusés par des impuretés créant un potentiel effectif aléatoire $V(x)$ au point $x$ dans l'approximation de liaison étroite (tight binding).

Cependant pour de nombreuses raisons il est intéressant de considérer le cas plus général d'opérateurs $H$ agissant sur $\ell^{2}(S)$, où $S$ est un ensemble dénombrable, définis par

$$
(H \psi)(x)=\sum_{y \in S} H(x, y) \psi(y)
$$

et où l'espace $\mathfrak{H}$ de tels opérateurs est muni d'une mesure de probabilité.

Outre l'intérêt mathématique évident d'étudier le cas général des opérateurs aux différences finies aléatoires, une telle généralisation est utile en physique pour inclure le cas du «désordre non diagonal» dans lequel des coefficients $H(x, y), x \neq y$ sont des variables aléatoires, pour inclure le cas où le réseau sous-jacent n'est pas $\mathbb{Z}^{v}$, mais un graphe n'ayant pas nécessairement de structure de groupe, comme dans les matériaux amorphes, ou bien enfin pour étudier des modèles plus compliqués nécessaires par exemple, dans l'approche des auteurs, à l'étude du seuil de mobilité.

Les questions d'intérêt dans l'étude des propriétés spectrales et des propriétés de transport associées à de tels opérateurs aléatoires sont principalement de trois sortes. La première question est celle de la nature de la densité d'état, en particulier de son support: présente-t-il des «gaps»... Ceci revient à déterminer le spectre de $H$, avec probabilité 1, puisque ce spectre est le support de la densité d'état. Un argument empirique a été présenté dans la référence [30] pour ce problème, dans le cas de l'équation de Schrödinger discrétisée.

La seconde question est la suivante: comment se comportent asymptotiquement dans l'espace les fonctions propres généralisées de $H$. Si elles décroissent

1 Notons cependant que de tels problèmes ont été posés dans la litérature mathématique bien avant les travaux d'Anderson sur les systèmes désordonnés: dès 1898, Stieltjes [29] conjecturait que (traduit en langage moderne) une équation de Schrödinger discrétisée unidimensionnelle avec un potentiel aléatoire possède avec probabilité 1 un ensemble de valeurs propres dense dans son spectre. (Nous remercions $\mathrm{F}$. Bentosela pour cette référence) 
suffisamment vite pour être de carré intégrable, alors les physiciens diront qu'elles sont localisées (en fait la plupart du temps, localisation sous-entend localisation exponentielle dans la littérature physique). Dans le cas contraire elles sont délocalisées. Grâce à une caractérisation des sous-espaces purement ponctuels et continus due à Ruelle $[26,1]$, en terme de l'évolution d'espace temps, nous pouvons identifier, le premier cas à une absence de diffusion du paquet d'onde alors que dans le second un paquet d'onde initialement localisé diffusera à l'infini. Ce problème revient donc à déterminer la nature du spectre de $H$ avec probabilité 1 . Il devrait être purement ponctuel dans le cas de la localisation, continu lorsque les fonctions propres sont délocalisées. Anderson ouvrit tout un domaine de recherche en arguant dans son article fondamental [2] qu'en dimension trois, pour un «désordre assez grand», tous les états de l'équation de Schrödinger avec un potentiel aléatoire sont localisés. Peu après Mott et Twose [20] montraient qu'en dimension un, tous les états devraient être localisés «aussi faible que soit le désordre».

Le troisième type de problème concerne les propriétés de transport de ces systèmes et dépend plus spécifiquement de l'interprétation physique que l'on choisit pour l'opérateur $H$. Si l'on cherche à décrire la diffusion d'électrons par des impuretés, on s'intéressera à la conductivité électrique, et en particulier on voudra déterminer si la conductivité statique est nulle ou non, différentiant ainsi un conducteur d'un isolant. Si l'on prend au contraire l'interprétation en oscillateurs harmoniques visant à décrire la dynamique d'un crystal dans l'approximation la plus simple possible, on s'intéressera au comportement de la conductivité thermique du système. Dans le cadre de la première interprétation, Mott et Twose [20] ont argué sous des conditions particulières dans le cas d'une équation de Schrödinger unidimensionnelle avec un potentiel aléatoire, que la conductivité électrique statique devrait être nulle. Halperin [12] conjectura que ce devrait être toujours le cas dans les systèmes unidimensionnels aussi faible que soit le désordre, et relia ce problème à celui de l'étude du déplacement moyen associé aux fonctions propres. Cet article a souvent été considéré, à tort, dans la littérature comme fournissant une preuve de l'absence de conductivité.

Bien entendu la majorité de ces problèmes restent soit incompris, soit à l'état de conjectures tant sur le plan de la physique que sur celui de la mathématique. Pour un état du sujet du point de vue de la physique, nous renvoyons à $[3,19,30]$. Quelques résultats ont été obtenus du point de vue de la mathématique, principalement basés sur un argument théorique mais non rigoureux dû à Borland [5]: il a introduit l'idée importante que pour des systèmes unidimensionnels, la localisation devrait être liée à une croissance exponentielle de la norme de l'image d'un vecteur par un produit aléatoire de matrices. La même année, Furstenberg [9] publiait sa très importante preuve de la positivité du plus grand exposant de Liapunov de produits de matrices aléatoires indépendantes sous des conditions appropriées. Ce résultat a été étendu au cas markovien, par Royer [25]. Il a été démontré par Matsuda et Ishii [17] et par Yoshioka [33] que les conditions d'application du théorème de Furstenberg sont remplies par les produits de matrices aléatoires associés à une équation de Schrödinger unidimensionnelle discrétisée avec un potentiel aléatoire lorsque celui-ci est constitué de variables aléatoires indépendantes. Dans ce cas, Casher et Lebowitz [7] ont montré 
l'absence de spectre absolument continu dans le cas de l'équation sur un demi-axe, et Pastur [23] dans le cas de l'axe complet, étendant de plus le résultat au cas markovien. Une étape importante a été franchie par Gold'sheid et al. [11] qui ont prouvé que le spectre de l'opérateur de Schrödinger avec un potentiel aléatoire est purement ponctuel dans diverses situations continues unidimensionnelles. De plus Molchanov [18] a pu montrer dans un cas que les fonctions propres sont exponentiellement décroissantes. Enfin concernant les propriétés de transport il a été prouvé par Casher et Lebowitz [7] que la conductivité thermique est infinie, dans un modèle de crystal harmonique désordonné unidimensionnel, et le comportement exact en a été obtenu par Verheggen [31]. Une revue des principaux travaux rigoureux avant 1973 sera trouvée dans [22, 13].

Les résultats que nous présentons dans ce papier répondent partiellement aux diverses questions posées ci-dessus. Certains d'entre eux ont été annoncés ailleurs [16]. Dans certaines situations nous pouvons déterminer exactement le spectre de $H$ (Sect. III). Nous établissons des critères généraux qui garantissent que le spectre de l'opérateur aléatoire $H$ est purement ponctuel ou continu (Sect. IV) ou que la conductivité électrique statique est nulle (Sect. V). Par ailleurs nous avons établi un formalisme général qui nous permet de calculer les quantités d'intérêt, les observables, comme limites d'intégrales multiples données par des formules explicites (Sect. VI). Appliquant enfin ces critères au cas de l'équation de Schrödinger unidimensionnelle, nous prouvons qu'avec probabilité 1, le spectre est purement ponctuel, les fonctions propres décroissent exponentiellement, et que la conductivité électrique statique est nulle (Sects. VII à IX). Nous obtenons par ailleurs diverses formules explicites pour des quantités utiles telles que la fonction spectrale moyenne et le rapport de participation.

L'intérêt de notre approche réside selon nous dans les faits suivants: bien que nous ne puissions conclure sur la nature du spectre actuellement que dans le cas unidimensionnel, notre approche est, dans son principe, indépendante de la dimension. En particulier elle transforme le problème original en une sorte de problème de spins continus sur un réseau et suggère une connection précise entre transition de phase en mécanique statistique et transition du spectre ponctuel au spectre continu. Cet aspect de notre travail est discuté dans la Sect. C.

\section{A. Le cas général}

\section{Définitions et préliminaires}

Au cours de cet article, nous considérons des opérateurs aux différences finies. Un cas particulier important en est l'opérateur de Schrödinger discrétisé. Nous définissons dans cette section la classe d'opérateurs aux différences finies que nous étudierons par la suite et indiquons quelques résultats préliminaires.

1) L'espace de base. Nous considérons un ensemble dénombrable de points $S$. Il peut être avantageusement pensé comme l'ensemble des sommets d'un graphe $G$ donné, souvent le réseau cubique $\mathbb{Z}^{d}$, mais aussi par exemple un arbre de Cayley, qui apparaît en physique dans le cas de l'approximation, dite de Bethe, des systèmes sur un réseau $\mathbb{Z}^{d}$. Dans ces cas, $S=S(G)$. 
Nous considérons l'espace des suites de carré sommable indexées par $S$ :

$$
\mathscr{H}=\ell_{\mathbb{C}, \mathbb{R}}^{2}(S)=\left\{\psi=\left.(\psi(x))_{x \in S}\left|\|\psi\|^{2}=\sum_{x \in S}\right| \psi(x)\right|^{2}<\infty\right\}
$$

où les suites sont à valeurs complexes ou réelles; étant donné en général l'absence d'ambiguités, nous laisserons implicite l'indice $\mathbb{C}$ ou $\mathbb{R}$. Nous utiliserons le produit scalaire $\langle$,$\rangle et la base hilbertienne \left\{\delta_{x}\right\}_{x \in S}$ naturellement associés:

$$
\begin{aligned}
\left\langle\psi, \psi^{\prime}\right\rangle & =\sum_{x \in S} \bar{\psi}(x) \psi^{\prime}(x) \\
\delta_{x}(y) & =\delta(x, y)
\end{aligned}
$$

où $\delta(x, y)$ est le symbole de Kronecker.

Nous considérons aussi l'espace

$$
f(S)=\left\{\psi=(\psi(x))_{x \in S} \mid \psi(x)=0 \text { pour tout } x \in S \text { sauf un nombre fini }\right\} .
$$

Nous introduisons aussi l'espace $\mathfrak{S}$ de fonctions symétriques sur $S \times S$, à valeurs réelles :

$$
\begin{aligned}
& \mathfrak{H}=\left\{H=(H(x, y))_{(x, y) \in S \times S} \mid \forall(x, y) \in S \times S, H(x, y)=H(y, x)\right. \\
& \text { et } \left.\|H\|_{1}^{\prime}=\operatorname{Sup}_{x \in S} \sum_{\substack{y \in S \\
y \neq x}}|H(x, y)|<\infty\right\} \text {. }
\end{aligned}
$$

Remarque. Dans la définition de l'espace $\mathfrak{H}$, il n'y a aucune contrainte sur les termes $H(x, x)$.

2) Les opérateurs. Soit $H \in \mathfrak{H}$. A cette fonction symétrique sur $S \times S$, nous associons les opérateurs $H_{N D}, \stackrel{\circ}{H}_{D}, \stackrel{\circ}{H}, H_{D}, H$ sur $\ell^{2}(S)$ définis par:

$$
\left(H_{N D} \psi\right)(x)=\sum_{\substack{y \in S \\ y \neq x}} H(x, y) \psi(y), \quad \forall x \in S
$$

qui est un opérateur auto-adjoint borné sur $\ell^{2}(S)$, de norme inférieure ou égale à $\|H\|_{1}^{\prime}\left(H_{N D}\right.$ est trivialement un opérateur borné de $\ell^{1}(S)$ dans lui-même et de $\ell^{\infty}(S)$ dans lui-même, et donc aussi de $\ell^{2}(S)$ dans lui-même, grâce au théorème d'interpolation de Riesz-Thorin qui assure aussi le résultat sur la norme).

$$
\left(\stackrel{\circ}{H}_{D} \psi\right)(x)=H(x, x) \psi(x), \quad \forall x \in S
$$

avec le domaine $D\left(\stackrel{\circ}{H}_{D}\right)=f(S)$,

avec le domaine $D(\stackrel{\circ}{H})=f(S)$.

$$
\stackrel{\circ}{H}=H_{N D}+\stackrel{\circ}{H}_{D}
$$

Les opérateurs $\stackrel{\circ}{H}_{D}$ et $\stackrel{\circ}{H}$ sont essentiellement auto-adjoints, et nous noterons $H_{D}$ et $H$ leurs fermetures qui sont auto-adjointes:

$$
\begin{aligned}
H & =H_{N D}+H_{D} \quad \text { de domaine } \\
D(H) & =\left\{\left.\psi \in \ell^{2}(S)\left|\sum_{x}\right| H(x, x) \psi(x)\right|^{2}<\infty\right\} .
\end{aligned}
$$

L'ensemble de ces opérateurs $H$, sera encore dénoté $\mathfrak{S}$. Ils admettent tous $f(S)$ pour coeur. 
La famille spectrale de l'opérateur $H$ sera notée $\left\{E_{H}(\lambda)\right\}$, et sa mesure spectrale $E_{H}(d \lambda)$

$$
\begin{aligned}
& E_{H}(\lambda)=\int_{-\infty}^{\lambda} E_{H}(d \lambda), \\
& E_{H}(A)=\int_{A} E_{H}(d \lambda),
\end{aligned}
$$

pour $A$ ensemble mesurable de $\mathbb{R}$. En l'absence d'ambiguité, la dépendance en $H$ sera oubliée et l'on écrira simplement $E(\lambda), E(d \lambda), E(A)$. Nous serons particulièrement amenés à considérer la mesure spectrale évaluée entre deux vecteurs de base: $\left\langle\delta_{x}, E_{H}(d \lambda) \delta_{y}\right\rangle$. Il s'agit d'une mesure réelle, et l'on peut considérer sa valeur absolue (voir par exemple [6]) qui sera dénotée

$$
\left|\left\langle\delta_{x}, E_{H}(d \lambda) \delta_{y}\right\rangle\right|
$$

Un exemple typique: l'opérateur de Schrödinger discrétisé

En choisissant:

$$
\begin{aligned}
S & =\mathbb{Z}^{v}, \quad H(x, x)=2 v+V(x), \\
H(x, y) & =-1 \quad \text { si } \quad d(x, y)=1, \\
H(x, y) & =0 \quad \text { si } \quad d(x, y)>1
\end{aligned}
$$

où $V(x)$ désigne le potentiel au point $x$ et $d(x, y)$ la distance sur $\mathbb{Z}^{v}$ telle que $d(0, x)$ $=\sum_{1 \leqq i \leqq v}\left|x^{(i)}\right|$, l'opérateur $H$ associé à la fonction $H(x, y)$ ci-dessus est l'opérateur de Schrödinger discrétisé avec le potentiel $V(x): H=-\Delta+V$.

3) Boites et limite thermodynamique. Soit $\Lambda$ un sous-ensemble fini de $S$, («une boîte») et $P^{\Lambda}$ l'opérateur de projection associé agissant sur $\ell^{2}(S)$ :

$$
\left(P^{\Lambda} \psi\right)(x)=\left\{\begin{array}{lll}
\psi(x) & \text { si } & x \in \Lambda \\
0 & \text { si } & x \in \Lambda^{c}=S \backslash \Lambda .
\end{array}\right.
$$

Puisque $S$ est dénombrable, et que $P^{\Lambda}$ agit sur $\ell^{2}(S)$, il existe toujours une suite $\left\{\Lambda_{i}\right\}_{i \in \mathbb{N}}$ de sous-ensembles finis de $S$ tels que

$$
s-\lim _{i \rightarrow \infty} P^{\Lambda_{i}}=I
$$

où $s$ - lim désigne la limite forte et $I$ l'opérateur identité. La limite selon une telle sous-suite est appelée limite thermodynamique et dans ce papier les boîtes $A$ et les limites $\Lambda$ croit vers $S: \Lambda \uparrow S$, seront toujours entendues au sens défini ci-dessus.

Il sera commode d'utiliser dans la suite:

$$
\begin{gathered}
\|\psi\|_{\Lambda}=\left\|P^{\Lambda} \psi\right\|, \\
\|\psi\|_{\Lambda^{c}}=\left\|\left(I-P^{\Lambda}\right) \psi\right\| .
\end{gathered}
$$

Considérons maintenant pour $H$ donné l'opérateur ${ }^{2}$

$$
H^{\Lambda}=P^{\Lambda} H P^{\Lambda}
$$

2 Nous avons choisi ici les conditions aux bords nulles pour la simplicité. On vérifie aussi facilement que tous nos résultats s'appliquent aussi au cas où $H^{4}$ serait construit à partir de conditions aux bords périodiques ou anti-périodiques qui sont utilisées parfois en physique. En effet on vérifie encore dans ce cas que $H^{A}$ converge fortement vers $H$ au sens de la résolvante 
qui est la restriction de l'opérateur $H$ à la région finie $\Lambda$ avec des conditions aux bords nulles ${ }^{3}$ (conditions de Dirichlet). On voit facilement que $H^{A}$ est un opérateur borné auto-adjoint; sa famille spectrale sera notée $E^{\Lambda}(\lambda)$.

Lorsque $\Lambda \uparrow S$, on voit facilement que $H^{\Lambda}$ tend vers $H$ fortement au sens de la résolvante ([24], Tome 1, Théorème VIII 25), ce qui assure la convergence des mesures spectrales $E^{\Lambda}(d \lambda)$ vers $E(d \lambda)$, au sens où pour tout $\psi \in \ell^{2}(S)$, la mesure $E^{\Lambda}(d \lambda) \psi$ converge faiblement (au sens des mesures) vers $E(d \lambda) \psi$.

Enfin considérant les valeurs absolues des mesures spectrales évaluées entre deux vecteurs de base, nous avons pour toute fonction $f$ continue, positive ou nulle:

$$
\int f(\lambda)\left|\left\langle\delta_{x}, E(d \lambda) \delta_{y}\right\rangle\right| \leqq \liminf _{\Lambda \uparrow S} \int f(\lambda)\left|\left\langle\delta_{x}, E^{\Lambda}(d \lambda) \delta_{y}\right\rangle\right|
$$

qui découle de la définition de la valeur absolue d'une mesure [6]. Il s'ensuit que si $A$ est un intervalle ouvert de $\mathbb{R}$

$$
\int_{A}\left\langle\delta_{x}, E(d \lambda) \delta_{y}\right\rangle\left|\leqq \liminf _{\Lambda \uparrow S} \int_{A}\right|\left\langle\delta_{x}, \mathrm{E}^{A}(\mathrm{~d} \lambda) \delta_{y}\right\rangle \mid .
$$

En effet $A$ étant ouvert

$$
\int_{A}\left|\left\langle\delta_{x}, E^{(A)}(d \lambda) \delta_{y}\right\rangle\right|=\lim _{n \rightarrow \infty} \int f_{n}(\lambda)\left|\left\langle\delta_{x}, E^{(\Lambda)}(d \lambda) \delta_{y}\right\rangle\right|
$$

où $f_{n}$ est une suite croissante de fonctions continues positives, convergeant ponctuellement vers la fonction caractéristique de $A$. Par ailleurs, la suite $f_{n}$ étant choisie croissante, pour tout $n$

$$
\int f_{n}(\lambda)\left|\left\langle\delta_{x}, E^{\Lambda}(d \lambda) \delta_{y}\right\rangle\right| \leqq \liminf _{\Lambda \uparrow S} \lim _{n \rightarrow \infty} \int f_{n}(\lambda)\left|\left\langle\delta_{x}, E^{\Lambda}(d \lambda) \delta_{y}\right\rangle\right| .
$$

4) Les mesures de probabilité. Considérons l'espace $\mathfrak{H}$ et munissons-le de la tribu des événements cylindriques. Nous introduisons des mesures de probabilité, notées $\mu$, sur cet espace mesurable. Si $f$ est une fonction mesurable sur $\mathfrak{h}, \mu(f)$ désignera sa valeur moyenne par rapport à $\mu$, et si $A$ est un sous-ensemble mesurable de $\mathfrak{H}, \mu(A)$ notera sa mesure. La probabilité conditionnelle, étant donné un événement $B$, sera désignée par $\mu(\cdot \mid B)$.

Nous pouvons alors énoncer la proposition suivante que nous utiliserons plusieurs fois dans la suite de ce texte et qui nous assure que «très souvent» l'opérateur $H^{A}$ n'a pas de valeurs propres dégénérées:

Proposition II.1. Soit $\mu$ une mesure de probabilité sur $\mathfrak{H}$. Supposons que la probabilité conditionnelle $\mu\left(H_{D}^{A} \mid H_{N D}^{A}\right)$ soit absolument continue par rapport à la mesure de Lebesgue. Alors avec probabilité 1, le spectre de $H^{A}$ est de multiplicité 1.

Preuve. En fait nous devons montrer que les valeurs propres d'une matrice symétrique sont non dégénérées pour presque toutes (au sens de la mesure de Lebesgue) les valeurs des éléments diagonaux. Or il est bien connu qu'une famille analytique de matrices a un nombre constant de valeurs propres distinctes, sauf sur un ensemble de mesure de Lebesgue nul [14]. Il suffit donc de constater que le

3 Cf. note en bas de la page précédente 
nombre de valeurs propres distinctes est égal à la dimension de l'espace lorsque les éléments diagonaux sont grands, mais d'ordres de grandeur différents, par rapport aux éléments non diagonaux.

\section{Détermination du spectre}

Nous nous restreindrons dans cette section au cas $S=\mathbb{Z}^{v}$. Des généralisations de nos résultats peuvent cependant s'obtenir lorsqu'il existe un groupe agissant sur $S$. Nous allons montrer qu'il est possible de déterminer complètement le spectre d'une large classe d'hamiltoniens stochastiques, avec probabilité 1 . Nous aurons ainsi obtenu le support de la densité d'état d'un grand nombre de modèles.

Dans ce qui suit $\mathrm{SpH}$ désigne le spectre de l'opérateur $H$. Alors

Théorème III.1. Soit $\mu$ une mesure de probabilité ergodique sur $\mathfrak{H}$. Alors $\mu$-presque tous les opérateurs $H$ ont même spectre.

Ce théorème découle directement du théorème ergodique, en utilisant le fait que $\mathrm{SpH}$ est un fermé. Il était déjà mentionné dans la référence [23].

Preuve. Soit $R_{n}$ un recouvrement dénombrable de $\mathbb{R}$ par des intervalles fermés $[a, b]$ d'extrémités rationnelles et de longueur $\frac{1}{n}: a, b \in \mathbb{Q}$ et $b-a=\frac{1}{n}$. Soit

$$
\mathfrak{H}_{a, b}=\{H \in \mathfrak{S} \mid \operatorname{Sp} H \cap[a, b] \neq \emptyset\} .
$$

Par l'hypothèse d'ergodicité de $\mu$, nous avons soit $\mu\left(\mathfrak{H}_{a, b}\right)=0$, soit $\mu\left(\mathfrak{H}_{a, b}\right)=1$. Soit maintenant

$$
\begin{aligned}
S_{n} & =\left\{[a, b] \in R_{n} \mid \mu\left(\mathfrak{H}_{a, b}\right)=1\right\} \\
\Sigma & =\bigcap_{n \geqq 1}\left\{\bigcup_{[a, b] \in S_{n}}[a, b]\right\} \\
\mathfrak{H}^{\prime} & =\bigcap_{n}\left\{\bigcap_{[a, b] \in S_{n}} \mathfrak{H}_{a, b}\right\} .
\end{aligned}
$$

$\mathfrak{S}^{\prime}$ est de mesure pleine et pour tout $H \in \mathfrak{S}^{\prime}$ nous avons $\operatorname{Sp} H=\Sigma$. En effet si $e \in \Sigma$, $e \in \bigcup_{[a, b] \in S_{n}}[a, b]$ pour tout $n$, et donc $\forall n$, dist $(\operatorname{Sp} H, e)<\frac{1}{n}$ quand $H \in \mathfrak{H}^{\prime}$, ce qui implique $\Sigma \subset \operatorname{Sp} H$. Par ailleurs si $\lambda \in \operatorname{Sp} H, H \in \mathfrak{H}^{\prime}$, alors $\lambda \in \bigcup_{[a, b] \in S_{n}}[a, b]$ pour tout $n$ et donc $\lambda \in \Sigma$, i.e. $\operatorname{Sp} H \subset \Sigma$.

Introduisons alors les supports suivants d'une mesure $\mu$ invariante par translation:

$$
\operatorname{Supp}_{D} \mu=\{\alpha \in \mathbb{R} \mid \forall \varepsilon, \mu(\{H \in \mathfrak{H},|H(x, x)-\alpha|<\varepsilon\})>0\}
$$

qui est le support de la distribution invariante du processus stationnaire $\{H(x, x)$; $\left.x \in \mathbb{Z}^{v}\right\}$, et

$$
\operatorname{Supp}_{R D} \mu=\{\alpha \in \mathbb{R} \mid \forall \varepsilon>0, \forall \Lambda, \mu(\{H \in \mathfrak{H}:|H(x, x)-\alpha|<\varepsilon, \forall x \in \Lambda\})>0\}
$$

où, dans (III.2), $\Lambda$ est l'ensemble des sites d'un cube de $\mathbb{Z}^{\nu}$.

Nous pouvons alors énoncer: 
Théorème III.2. Soit $\mu$ une mesure de probabilité ergodique sur $\mathfrak{H}$. Alors pour $\mu$-presque tout $H$ :
a) $\operatorname{Sp} H \supset \mathrm{Sp}_{N D}+\operatorname{Supp}_{R D} \mu$
b) ${ }^{4} \operatorname{Sp} H \subset\left[\operatorname{Inf} \operatorname{SpH}_{N D}, \operatorname{SupSpH} H_{N D}\right]+\operatorname{Supp}_{D} \mu$.

Remarquons que $\mathrm{Sp} H_{N D}$ est en fait constant avec probabilité 1 (cela suit du Théorème III.1). Le spectre de $H$ est donc exactement déterminé avec probabilité 1 , dès que $\alpha) \operatorname{Sp} H_{N D}=\left[\operatorname{Inf} \operatorname{Sp} H_{N D}, \operatorname{SupSp} H_{N D}\right.$, ce qui est par exemple le cas si $H_{N D}$ est la partie non diagonale de l'opérateur de Laplace discrétisé, et $\beta$ ) $\operatorname{Supp}_{R D} \mu$ $=\operatorname{Supp}_{D} \mu$ ce qui est vérifié dans la majorité des cas d'intérêt, en particulier dans le cas des mesures produit, mais plus généralement dans le cas de mesures de Gibbs ergodiques et sans conditions d'exclusion. Nous singularisons le cas particulier suivant pour son intérêt physique:

Corollaire III.3. Soit l'opérateur de Schrödinger discrétisé sur $\mathbb{Z}^{v}, H(V)=-\Delta+V$, où le potentiel $V$ est un ensemble $\{V(x)\}_{x \in \mathbb{Z}^{v}}$ de variables aléatoires indépendantes de même loi de probabilité $\varrho$. Alors: pour presque tout $V, \mathrm{Sp} H=[0,4 v]+\operatorname{Supp} \varrho$ où Supp @ désigne le support de la loi @.

Preuve du théorème III.2. Prouvons d'abord la partie a) du théorème. Il suit du Théorème III.1 qu'il existe un sous-ensemble $\mathfrak{H}^{\prime}$ de $\mathfrak{H}$, de mesure pleine, invariant par les translations, sur lequel le spectre de $H_{N D}$ est constant. Considérons $H \in \mathfrak{S}^{\prime}$, et $\alpha \in \mathrm{Sp} H_{N D}$, ainsi que $\beta \in \operatorname{Supp}_{R D} \mu$. Par le critère de Weyl ([24], Vol. 1, p. 264), il existe une suite $\psi_{n}, \psi_{n} \in \ell^{2}\left(\mathbb{Z}^{v}\right),\left\|\psi_{n}\right\|=1$, telle que:

$$
\lim _{n \rightarrow \infty}\left\|\left(H_{N D}-\alpha\right) \psi_{n}\right\|=0
$$

et comme $H_{N D}$ est un opérateur borné, $\psi_{n}$ peut en fait être choisi dans $f(S)$. Associons alors à $H$ une suite de cubes de $\mathbb{Z}^{v}, \Lambda_{n}(H, \alpha)$, telle que

$$
\psi_{n}(x)=0 \quad \text { si } \quad x \notin \Lambda_{n}(H, \alpha) .
$$

Nous allons montrer qu'avec probabilité $1, \lambda=\alpha+\beta$ est dans le spectre de $H$. Pour cela, définissons l'événement:

$$
A_{n}(\alpha, \beta)=\left\{H \in \mathfrak{Y}, H \in \mathfrak{S}^{\prime}\left|\quad \forall x \in \Lambda_{n}(H, \alpha),\right| H(x, x)-\beta \mid<\frac{1}{n}\right\} .
$$

Puisque $\beta \in \operatorname{Supp}_{R D} \mu$ et que $\mu\left(\mathfrak{H}^{\prime}\right)=1, \mu\left(A_{n}(\alpha, \beta)\right)>0$. Il y a clairement une méthode de choix systématique des cubes $\Lambda_{n}(H, \alpha)$ qui fasse de chaque $A_{n}(\alpha, \beta)$ un événement invariant par translation. Par conséquent $\mu\left(A_{n}(\alpha, \beta)\right)=1$ et donc aussi:

$$
\mu\left(\bigcap_{n \geqq 1} A_{n}(\alpha, \beta)\right)=1 .
$$

Mais pour $H \in \bigcap_{n \geqq 1} A_{n}(\alpha, \beta)$, nous avons:

$$
\forall_{n},\left\|(H-\lambda) \psi_{n}\right\| \leqq\left\|\left(H_{N D}-\alpha\right) \psi_{n}\right\|+\left\|\left(H_{D}-\beta\right) \psi_{n}\right\|
$$

4 Si $A$ et $B$ sont deux sous-ensembles de $\mathbb{R}$,

$$
A+B=\{\lambda \in \mathbb{R} \mid \lambda=\alpha+\beta, \alpha \in A, \beta \in B\}
$$


et

$$
\lim _{n \rightarrow \infty}\left\|(H-\lambda) \psi_{n}\right\|=0
$$

où nous avons utilisé (III.3)-(III.5). En utilisant le critère de Weyl, au sens réciproque, ceci assure que $\lambda=\alpha+\beta \in \mathrm{Sp} H$.

Enfin, si $A$ est un sous-ensemble dénombrable dense de $\operatorname{Sp} H_{N D}$ quand $H \in \mathfrak{S}^{\prime}$ et $B$ un sous-ensemble dénombrable dense de $\operatorname{Supp}_{R D} \mu$, comme

$$
\mu\left(\bigcap_{\alpha \in A} \bigcap_{\beta \in B} \bigcap_{n \geqq 1} A_{n}(\alpha, \beta)\right)=1
$$

avec probabilité 1 , tout $\lambda=\alpha+\beta, \alpha \in A, \beta \in B$ appartient au spectre de $H$, ce qui achève la preuve de la partie a) du théorème puisque $\operatorname{Sp} H$ est un fermé.

Venons à la preuve de la partie b). Soit $\mathfrak{H}^{\prime \prime}$ défini par

$$
\mathfrak{H}^{\prime \prime}=\left\{H \in \mathfrak{S} \mid \operatorname{Sp} H_{D}=\operatorname{Supp}_{D} \mu\right\} \text {. }
$$

On voit facilement que $\mu\left(\mathfrak{S}^{\prime \prime}\right)=1$ et donc aussi

$$
\mu\left(\mathfrak{H}^{\prime} \cap \mathfrak{H}^{\prime \prime}\right)=1
$$

Soit alors $H \in \mathfrak{H}^{\prime} \cap \mathfrak{H}^{\prime \prime}$. Le résultat suit de la propriété suivante ([14], Chap. V, Théorème 4.10 , p. 291)

$$
\operatorname{dist}\{\operatorname{Sp}(U+V), \operatorname{Sp} V\} \leqq\|U\|
$$

appliquée à $U=H_{N D}-\frac{1}{2}\left(\operatorname{Sup} \operatorname{Sp} H_{N D}-\operatorname{Inf} \operatorname{Sp} H_{N D}\right) I$, et $V=H_{D}+\frac{1}{2}\left(\operatorname{Sup} \operatorname{Sp} H_{N D}\right.$ - Inf $\left.\mathrm{Sp} H_{N D}\right) I$, où $I$ désigne l'identité.

Diverses études avaient porté, dans la littérature physique, sur le spectre de tels opérateurs aléatoires. Des résultats du type de la partie b) de notre Théorème III.2 avaient été obtenus par Weaire et Thorpe [32]; une forme du Corollaire III.3 était mentionnée par Thouless [30] justifiée par l'argument physique sur lequel repose notre démonstration.

\section{Critères spectraux}

Nous étudions dans cette section la nature du spectre des opérateurs $H$. Nous indiquons d'abord quelques résultats qui découlent de ceux de la Sect. III, puis des conséquences faciles mais utiles et physiquement importantes du théorème ergodique. Ces résultats (Théorèmes IV.1 et IV.2) sont décrits ici dans le cas où $S=\mathbb{Z}^{v}$, des extensions existent cependant aussi dans le cas où il existe un groupe agissant sur $S$. Nous montrerons que la nature du spectre (purement ponctuel, continu...) ne change pas, avec probabilité 1 . De ce fait, le seuil de mobilité, s'il existe, séparant le spectre continu du spectre ponctuel, est constant avec probabilité 1. Dans les théorèmes suivants, qui n'exigent aucune condition sur $S$, nous construisons des critères permettant de déterminer la nature du spectre de $H$.

Proposition IV.1. Soit $\mu$ une mesure de probabilité ergodique sur $\mathfrak{H}\left(\mathbb{Z}^{\nu}\right)$, telle que pour $\mu$-presque tout $H: \mathrm{Sp} H_{N D}=\left[\operatorname{Inf} \operatorname{Sp} H_{N D}\right.$, Sup Sp $\left.H_{N D}\right]$, non réduit à un point. Alors $\mu$-presque tout opérateur $H$ n'a pas de spectre discret et plus généralement pas de valeur propre isolée. 
Ce résultat est une conséquence immédiate du Théorème III.2. Il s'applique en particulier à l'opérateur de Schrödinger discrétisé.

Théorème IV.2. Soit $\mu$ une mesure de probabilité ergodique sur $\mathfrak{H}\left(\mathbb{Z}^{v}\right)$. Alors:

a) $\forall \alpha, \beta \in \mathbb{R}$, avec probabilité 1 , la nature du spectre de $H$ dans l'intervalle $(\alpha, \beta)$, est la même.

b) $\forall \lambda \in \mathbb{R}$, avec probabilité 1 , soit $\lambda$ n'est pas valeur propre de $H$, soit $\lambda$ est valeur propre et de dimension infinie.

La partie b) de ce théorème était dérivée dans [23] dans l'étude de l'opérateur de Schrödinger discrétisé, avec un potentiel aléatoire, à une dimension. Il y était conclu que dans ce cas un réel $\lambda$ donné, avec probabilité 1 n'est pas valeur propre. Une conséquence analogue se déduit de notre résultat si $\mu$-presque tout opérateur $H$ ne peut admettre que des valeurs propres de dimension finies (par exemple, l'opérateur de Schrödinger aux différences finies dans une bande).

La partie a) montre un résultat espéré physiquement: pour un intervalle d'énergie donné, le comportement du système (localisation, diffusion...) sera le même pour presque toute réalisation, si la mesure est ergodique. Il implique aussi que s'il existe un seuil de mobilité, celui-ci est constant avec probabilité 1.

Preuve. La partie a) du théorème se déduit ainsi : avec probabilité 1 soit le spectre est extérieur à l'intervalle $(\alpha, \beta)$, soit il l'intersecte (Théorème III.1). Dans ce dernier cas, considérons les événements possibles: le spectre dans l'intervalle $(\alpha, \beta)$ peut être purement ponctuel, singulier continu, absolument continu, ou bien présenter deux de ces caratères ou enfin les trois. Ces sept événements sont disjoints et exhaustifs. Par ailleurs, ils sont invariants par translation et mesurables (Lemme IV.3, ciaprès) et donc, par l'ergodicité, de mesure nulle ou pleine. Nécessairement l'un d'entre eux est donc de mesure 1, les autres de mesure nulle.

Que $\lambda$ soit valeur propre avec probabilité 0 ou 1 , dans la partie b) du théorème, se déduit de façon analogue. Qu'elle ne puisse qu'être de dimension infinie se déduit comme chez Pastur [23]: on remarque d'abord que si $\lambda$ est valeur propre de $\mu$-presque tout $H$, nécessairement il existe $x \in S$ tel que $\mu\left(\left(\delta_{x}, E_{H}(\{\lambda\}) \delta_{x}\right)\right)>0$, où $E_{H}(\{\lambda\})$ désigne le projecteur sur la valeur propre $\lambda$ associé à l'opérateur $H$. Sinon on en déduirait que pour $\mu$-presque tout $H, E_{H}(\{\lambda\})$ serait l'opérateur nul. Or par ailleurs :

$$
\operatorname{dim} E_{H}(\{\lambda\}) \geqq \sum_{x \in S}\left(\delta_{x}, E_{H}(\{\lambda\}) \delta_{x}\right)
$$

et nous voyons que $\operatorname{dim} E_{H}(\{\lambda\})$, qui est égale à $\mu\left(\operatorname{dim} E_{H}(\{\lambda\})\right)$ encore par le théorème ergodique, est infinie puisque $\mu\left(\left(\delta_{x}, E_{H}(\{\lambda\}) \delta_{x}\right)>0\right.$, indépendemment de $x$ par l'invariance par translation.

Il nous reste donc à montrer maintenant le lemme de mesurabilité utilisé cidessus.

Lemme IV.3. Les sept événements: "Le spectre de $H$ dans l'intervalle $(\alpha, \beta)$ est purement ponctuel, absolument continu, singulier continu, a deux de ces caractères, ou les trois» sont des événements mesurables. 
Preuve $^{5}$ : Notons d'abord que l'application qui a $H \in \mathfrak{H}, \mathfrak{H}$ muni de la topologie de la convergence forte sur $f(S)$ fait correspondre la mesure

$$
v(H)=\sum_{n \in \mathbb{N}} \frac{1}{2^{n}}\left\langle\delta_{x_{n}}, E(\lambda) \delta_{x_{n}}\right\rangle
$$

où la suite $\left\{x_{n}\right\}_{n \in \mathbb{N}}$ décrit $S$, est une application continue, si l'on munit les mesures de la convergence vague. Il suffira donc de montrer la mesurabilité des ensembles de mesures satisfaisant telle ou telle propriété dans l'intervalle $A=(\alpha, \beta)$. Considérons d'abord l'ensemble $\mathscr{M}_{c}$ des mesures continues dans $A$. Elles sont caractérisées par

$$
v \otimes v(\Delta)=0
$$

où $\Delta$ désigne la diagonale de $A \times A . \mathscr{M}_{c}$ est donc mesurable.

Soit maintenant $\mathscr{M}_{a c}$ l'ensemble des mesures absolument continues dans $A$. $\mathscr{M}_{a c}$ est l'image continue, injective de l'espace polonais $L^{1}(A)$ dans l'espace $\mathscr{M}$ des mesures de Radon sur $A$. Donc $\mathscr{M}_{a c}$ est un borélien.

Soit alors l'image de $\left(\mathscr{M}_{a c} \backslash\{0\}\right) \times\left(\mathscr{M}_{c} \backslash \mathscr{M}_{a c}\right)$ dans $\mathscr{M}_{c}$ par l'application somme, qui est continue et injective: l'ensemble des mesures continues, non purement absolument continues, est donc mesurable, de même que son complémentaire dans $\mathscr{M}_{c}, \mathscr{M}_{s c}$ l'ensemble des mesures singulières continues dans $A$.

Considérons encore le cas de $\mathscr{M}_{p p}$, l'ensemble des mesures purement ponctuelles dans $A$. Il est l'image continue (non injective) de l'espace polonais $A^{\mathbb{N}} \times \ell^{1}$ dans $\mathscr{M}$ par l'application:

$$
\left(a_{i} \in A, x_{i} \in \mathbb{R}^{+}\right)_{i \in \mathbb{R}} \rightarrow \sum_{i \in \mathbb{N}} a_{i} \delta_{x_{i}}
$$

et est donc un ensemble souslinien (suslin set).

Les derniers cas se traitent dans le même esprit.

Nous abordons maintenant la construction de critères permettant de déterminer la nature du spectre de $H$ et des fonctions propres. Nous revenons maintenant au cas général d'un espace $S$ dénombrable. Nous étudions d'abord le cas du spectre purement ponctuel. Mais auparavant nous introduisons les

Définitions. Soit un opérateur $H, H \in \mathfrak{H}$. Nous noterons $\varrho_{H}(x, y ; d \lambda)$ la mesure spectrale de $H$ entre les points $x$ et $y$ de $S$ et nous appellerons mesure de corrélation de $H$ entre les points $x$ et $y$ de $S$ sa valeur absolue $\bar{\varrho}_{H}(x, y ; d \lambda)$ :

$$
\begin{gathered}
\varrho_{H}(x, y ; d \lambda)=\left\langle\delta_{x}, E_{H}(d \lambda) \delta_{y}\right\rangle, \\
\bar{\varrho}_{H}(x, y ; d \lambda)=\left|\left\langle\delta_{x}, E_{H}(d \lambda) \delta_{y}\right\rangle\right| .
\end{gathered}
$$

La mesure d'un intervalle $A$ de $\mathbb{R}$ par rapport à ces deux mesures sera donc $\varrho_{H}(x, y ; A)$ et $\bar{\varrho}_{H}(x, y ; A)$.

Soit $\mu$ une mesure de probabilité sur $\mathfrak{H}$. Nous noterons $\varrho_{\mu}(x, y ; d \lambda)$ la mesure spectrale moyenne entre les points $x$ et $y$ de $S$ et appellerons mesure de corrélation de

5 Pour les définitions ou les résultats utilisés dans ce lemme, nous renvoyons à la référence [28] 
$\mu$ entre les points $x$ et $y$ de $S, \bar{\varrho}_{\mu}(x, y ; d \lambda)$, obtenues en moyennant ${ }^{6}$ en $\mu$ les mesures $\varrho_{H}$ et $\bar{\varrho}_{H}$.

En l'absence d'ambiguité, les indices $H$ ou $\mu$ seront supprimés et nous écrirons $\varrho(x, y ; d \lambda), \ldots$ L'indice $\Lambda$ repérera les quantités analogues calculées avec $H^{\Lambda}$, et nous aurons ainsi $\varrho^{\Lambda}(x, y ; d \lambda), \varrho_{\mu}^{\Lambda}(x, y ; A) \ldots$

En particulier, lorsque $H^{\Lambda}$ n'a pas de valeurs propres dégénérées ${ }^{7}$ dans l'intervalle $A, \varrho^{\Lambda}$ et $\varrho^{\Lambda}$ peuvent s'écrire

$$
\begin{aligned}
& \varrho_{H}^{A}(x, y ; A)=\sum_{\lambda \in \operatorname{Sp} H^{\Lambda} \cap A} \psi_{\lambda}(x) \psi_{\lambda}(y), \\
& \varrho_{H}^{A}(x, y ; A)=\sum_{\lambda \in \operatorname{Sp} H^{\Lambda} \cap A}\left|\psi_{\lambda}(x) \psi_{\lambda}(y)\right|
\end{aligned}
$$

où $\psi_{\lambda}$ désigne la fonction propre de $H^{\Lambda}$ associée à la valeur propre $\lambda$.

Nous pouvons alors énoncer:

Théorème IV.4. Soit un opérateur $H, H \in \mathfrak{S}(S)$ et $A \subset \mathbb{R}$ un intervalle ouvert. Supposons que $H$ satisfait l'une des conditions suivantes:
a) $\forall x \in S, \sum_{y \in S}\left(\bar{\varrho}_{H}(x, y ; A)\right)^{2}<\infty$,
b) $\forall x \in S, \sum_{y \in S} \bar{\varrho}_{H}(x, y ; A)<\infty$,
c) $\forall x \in S, \liminf _{\Lambda \uparrow S} \sum_{y \in S} \bar{\varrho}_{H}^{A}(x, y ; A)<\infty$.

Alors le spectre de l'opérateur $H$, dans A, est purement ponctuel.

Preuve. Remarquons d'abord que la condition c) implique b). En effet (II.19) s'écrit:

$$
\bar{\varrho}_{H}(x, y ; A) \leqq \liminf _{\Lambda \uparrow S} \bar{\varrho}_{H}^{\Lambda}(x, y ; A)
$$

et par le lemme de Fatou

$$
\sum_{x \in S} \liminf _{\Lambda \uparrow S} \bar{\varrho}_{H}^{A}(x, y ; A) \leqq \liminf _{\Lambda \uparrow S} \sum_{x \in S} \bar{\varrho}_{H}^{A}(x, y ; A) .
$$

D'autre part la condition b) implique trivialement a). Il nous reste donc à montrer que (IV.9) implique que le spectre de $H$ est purement ponctuel dans $A$. Le sous espace purement ponctuel $\mathscr{H}_{p p}(H)$ de $\mathscr{H}=\ell^{2}(S)$ associé à $H$ étant fermé, il suffit de montrer que $E(A) \delta_{x}$ est dans $\mathscr{H}_{p p}(H)$ pour tout $x \in S ; E(A)$ désigne le projecteur sur l'intervalle spectral $A$. Selon un critère obtenu par Ruelle [26] et étendu par Amrein et Georgescu [1] ceci revient à prouver

$$
\forall x \in S, \lim _{\Lambda \uparrow S} \operatorname{Sup}_{t \in \mathbb{R}}\left\|P^{\Lambda^{c}} e^{-i t H} E(A) \delta_{x}\right\|^{2}=0
$$

6 On vérifie facilement que les mesures $\varrho_{H}$ et $\bar{\varrho}_{H}$ sont $\mu$ mesurables

7 Ce qui est «souvent» réalisé; voir par exemple notre Proposition II.1 
(dans notre cas, ce critère est obtenu par le théorème p. 642 [1] en y choisissant $\left.S_{n}=E([-n,+n])\right)$. Mais (IV.14) se réécrit

$$
\begin{aligned}
& \lim _{\Lambda \uparrow S} \operatorname{Sup}_{t \in \mathbb{R}} \sum_{y \in \Lambda^{c}}\left|\left\langle\delta_{y}, \mathrm{e}^{-i t H} \mathrm{E}(\mathrm{A}) \delta_{x}\right\rangle\right|^{2}, \\
& =\lim _{\Lambda \uparrow S} \operatorname{Sup}_{t \in \mathbb{R}^{\mathbb{R}}} \sum_{y \in \Lambda^{c}}\left|\int_{A} \mathrm{e}^{-i t \lambda}\left\langle\delta_{y}, \mathrm{E}(\mathrm{d} \lambda) \delta_{x}\right\rangle\right|^{2}, \\
& \leqq \lim _{\Lambda \uparrow S} \sum_{y \in \Lambda^{c}}\left(\bar{\varrho}_{H}(x, y ; A)\right)^{2},
\end{aligned}
$$

qui est nul pour tout $x \in S$ si (IV.9) est satisfait.

En fait, nous sommes intéressés à prouver des propriétés spectrales d'opérateurs aléatoires avec probabilité 1. Mais il est plus facile d'étudier des valeurs moyennes, et même, nous le verrons, des valeurs moyennes de grandeurs définies à partir de l'hamiltonien restreint à une grande boîte $\Lambda$. Le corollaire cidessous établit un critère plus facilement utilisable.

Corollaire IV.5. Soit $\mu$ une mesure de probabilité sur $\mathfrak{H}(S)$. Supposons que $\mu$ satisfait l'une des conditions suivantes:

$$
\begin{aligned}
& \text { a) } \forall x \in S, \sum_{y \in S} \mu\left\{\left(\bar{\varrho}_{H}(x, y ; A)\right)^{2}\right\}<\infty, \\
& \text { b) } \forall x \in S, \sum_{y \in S} \bar{\varrho}_{\mu}(x, y ; A)<\infty, \\
& \text { c) } \forall x \in S, \liminf _{\Lambda \uparrow S} \sum_{y \in S} \bar{\varrho}_{\mu}^{\Lambda}(x, y ; A)<\infty .
\end{aligned}
$$

Alors le spectre de $\mu$-presque tout $H$ est purement ponctuel dans l'intervalle ouvert $A$ et pour $\mu$-presque tout $H$ les projecteurs associés aux valeurs propres $\lambda, \lambda \in A$ satisfont la propriété de décroissance

$$
\begin{aligned}
\left|\left\langle\delta_{x}, E_{H}(\{\lambda\}) \delta_{y}\right\rangle\right| & \leqq C(H, \mu, x) f(y)^{-1} \bar{\varrho}_{\mu}(x, y ; A) \\
& \leqq C(H, \mu, x) f(y)^{-1} \liminf _{\Lambda \uparrow S} \bar{\varrho}_{\mu}^{-\Lambda}(x, y ; A)
\end{aligned}
$$
où $f(y)$ est une fonction réelle arbitraire satisfaisant $f(y)>0, \sum_{y \in S} f(y)=1$, et
$C(H, \mu, x)$ est une constante.

Preuve. Prouvons par exemple le résultat sous l'hypothèse $c$ ). Nous avons pour tout $x \in S$ :

$$
\begin{aligned}
\liminf _{\Lambda \uparrow S} \sum_{y \in S} \bar{\varrho}_{\mu}^{\Lambda}(x, y ; A) & =\liminf _{\Lambda \uparrow S} \mu\left(\sum_{y \in S} \bar{\varrho}_{H}^{\Lambda}(x, y ; A)\right) \\
& \geqq \mu\left(\underset{\Lambda \uparrow S}{\liminf } \sum_{y \in S} \bar{\varrho}_{H}^{\Lambda}(x, y ; A)\right) .
\end{aligned}
$$

Donc la condition $c$ ) assure que pour tout $x \in S$, il existe un ensemble de $\mu$-mesure pleine, tel que $\liminf _{\Lambda \uparrow S} \sum_{y \in S} \bar{\varrho}_{H}^{\Lambda}(x, y ; A)<\infty$. 
Comme les intersections dénombrables d'ensembles de mesure 1 sont encore de mesure pleine, et utilisant le Théorème IV.4, nous obtenons le résultat sur la nature du spectre.

La propriété de décroissance des projecteurs s'obtient par

$$
\mu\left(\sum_{y \in S} \bar{\varrho}_{\mu}(x, y ; A)^{-1} \bar{\varrho}_{H}(x, y ; A) f(y)\right) \equiv \sum_{y \in S} f(y)=1 .
$$

De cette identité se déduit avec probabilité 1

$$
\bar{\varrho}_{H}(x, y ; A) \leqq C(H, \mu, x) f(y)^{-1} \bar{\varrho}_{\mu}(x, y ; A) .
$$

Remarques. a) ces critères, Théorème IV.4, Corollaire IV.5, semblent apparaître ici pour la première fois, tant du point de vue de la littérature physique que mathématique. Ils seront utilisés sous la forme du Corollaire IV.5c) dans la Sect. IX de cet article pour prouver que l'opérateur de Schrödinger discrétisé en dimension $d=1$, avec un potentiel aléatoire a un spectre purement ponctuel pour presque tout potentiel, du moins sous certaines hypothèses.

b) Il est d'intérêt de noter que par example si $H$ est le Laplacien discrétisé, $\bar{\varrho}_{H}(x, y ; A)$ est non seulement non intégrable, mais ne tend pas vers 0 à l'infini. Ceci est-il une caractéristique de l'existence d'une composante continue dans le spectre? En fait, ces critères sont fortement reminiscents de la situation en mécanique statistique, où l'intégrabilité de la fonction de corrélation à deux points est associée à l'absence de transition de phase. On pourrait de plus, conjecturer que si $\varrho_{\mu}(x, y ; A)$ ne tend pas vers 0 quand $|x-y| \rightarrow \infty$ alors le spectre dans $A$ est absolument continu. Nous reviendrons plus loin sur ces relations avec la mécanique statistique.

Nous étudions maintenant le spectre continu. Pour cela nous introduisons les

Définitions. Soit un opérateur $H, H \in \mathfrak{H}$. Nous appellerons mesure de transfert ${ }^{8}$ de $H$ entre les points $x$ et $y$ de $S$, la mesure

$$
R_{H}(x, y ; d \lambda)=\int\left(\left\langle\delta_{x}, E_{H}(d \lambda) \delta_{y}\right\rangle \otimes\left\langle\delta_{x}, E_{H}\left(d \lambda^{\prime}\right) \delta_{y}\right\rangle\right) \cdot \delta_{\lambda}\left(\lambda^{\prime}\right) d \lambda^{\prime}
$$

en particulier $R_{H}(x, x ; d \lambda)$ sera appelée mesure de récurrence ${ }^{8}$ de $H$ au point $x$. $R_{H}(x, y ; A)$ désignera la mesure de l'intervalle $A$ selon la mesure de transfert et l'on aura donc

$$
R_{H}(x, y ; A)=\int_{A} R_{H}(x, y ; d \lambda)=\iint_{\Delta_{A}}\left\langle\delta_{x}, E_{H}(d \lambda) \delta_{y}\right\rangle \otimes\left\langle\delta_{x}, E_{H}\left(d \lambda^{\prime}\right) \delta_{y}\right\rangle
$$

où $\Delta_{A}$ désigne la diagonale de $A \times A$.

Soit $\mu$ une mesure de probabilité sur $\mathfrak{H}$. La moyenne ${ }^{9}$ en $\mu$ de $R_{H}(x, y ; d \lambda)$ sera notée $R_{\mu}(x, y ; d \lambda)$, la mesure de transfert ${ }^{8}$ de $\mu$ entre les points $x$ et $y$. En particulier $R_{\mu}(x, x ; d \lambda)$ est la mesure de récurrence de $\mu$ en $x$.

8 Ces dénominations sont justifiées par le fait que, dans l'interprétation quantique où des fonctions d'ondes évoluent dans le temps selon l'opérateur $\exp \{-$ it $H\}, R_{H}(x, y ; A)$ est la contribution des énergies dans l'intervalle $A$ à la probabilité, moyennée dans le temps, pour qu'une particule soit en $y$ si elle était à l'instant initial localisée en $x$. Le cas $y=x$ correspond à la probabilité de retourner au point $x$ de départ

9 On voit facilement que $R_{H}$ est $\mu$-mesurable 
En l'absence d'ambiguités, les indices $H$ ou $\mu$ seront supprimés. L'indice $\Lambda$ indexera les quantités analogues construites à partir de $H^{\Lambda}$, et nous aurons $R_{H}^{\Lambda}(x, y ; d \lambda), R_{\mu}^{\Lambda}(x, x ; A) \ldots$

En particulier lorsque $H^{\Lambda}$ n'a pas de valeurs propres dégénérées ${ }^{10}$ dans l'intervalle $A, R_{H}^{A}(x, y ; A)$ s'écrit

$$
R_{H}^{A}(x, y ; A)=\sum_{\lambda \in \operatorname{Sp} H^{\Lambda} \cap A} \psi_{\lambda}(x)^{2} \psi_{\lambda}(y)^{2}
$$

où $\psi_{\lambda}$ désigne la fonction propre de $H^{\Lambda}$ associée à la valeur propre $\lambda$.

Nous devons introduire aussi la quantité $R_{H}^{A}(x, y ; A, \varepsilon)$ et sa $\mu$-moyenne $R_{\mu}^{\Lambda}(x, y ; A, \varepsilon)$ par

$$
R_{H}^{\Lambda}(x, y ; A, \varepsilon)=\iint_{\Delta_{A, \varepsilon}}\left\langle\delta_{x}, E_{H}^{A}(d \lambda) \delta_{y}\right\rangle \otimes\left\langle\delta_{x}, E_{H}^{A}\left(d \lambda^{\prime}\right) \delta_{y}\right\rangle
$$

où

$$
\Delta_{A, \varepsilon}=\{A \times A\} \cap\left\{\left(\lambda, \lambda^{\prime}\right):\left|\lambda^{\prime}-\lambda\right|<\varepsilon\right\} .
$$

Si $H^{\Lambda}$ n'a pas de valeurs propres dégénérées dans l'intervalle $A$, cette quantité s'écrit aussi

$$
R_{H}^{\Lambda}(x, y ; A, \varepsilon)=\sum_{\substack{\lambda, \lambda^{\prime} \in \mathbf{S p} H^{\Lambda} \cap \cap A \\\left|\lambda-\lambda^{\prime}\right|<\varepsilon}} \psi_{\lambda}(x) \psi_{\lambda}(y) \psi_{\lambda^{\prime}}(x) \psi_{\lambda^{\prime}}(y) .
$$

Nous pouvons maintenant énoncer le

Théorème IV.6. Soit un opérateur $H, H \in \mathfrak{S}(S)$ et soit $A \subset \mathbb{R}$ un intervalle ouvert.

a) Une condition nécessaire pour que le spectre de $H$ dans $A$ soit purement continu est que

$$
\forall x \in S, \lim _{\Lambda \uparrow S} R_{H}^{A}(x, x ; A)=0 .
$$

b) Une condition nécessaire et suffisante pour que le spectre de $H$ dans $A$ soit purement continu est que

$$
\forall x \in S, R_{H}(x, x ; A)=\lim _{\in \rightarrow 0} \lim _{\Lambda \uparrow S} R_{H}^{A}(x, x ; A, \varepsilon)=0 .
$$

Preuve. Remarquons d'abord que la première égalité de (IV.34) se déduit directement de la convergence des mesures spectrales: cette convergence, assure celle des produits tensoriels apparaissant dans les définitions des $R_{H}^{A}$, et donc de la mesure des ouverts $\Delta_{A, \varepsilon}$, et d'autre part, on a $\Delta_{A}=\bigcap_{\varepsilon} \Delta_{A, \varepsilon^{\prime}}$. De plus, on a aussi facilement au vu de la convergence des mesures spectrales que

$$
\lim _{\Lambda \uparrow S} R_{H}^{\Lambda}(x, x ; A) \leqq R_{H}(x, x ; A)
$$

parce que

$$
R_{H}^{A}(x, x ; A)=\operatorname{Inf}_{\varepsilon>0} R_{H}^{A}(x, x ; A, \varepsilon)
$$

10 Ce qui est «souvent» réalisé : voir par exemple notre Proposition II.1 
L'inégalité (IV.35) assure la proposition a) du théorème ci-dessus à partir de la proposition b). Il ne reste donc plus à prouver que $R_{H}(x, x ; A)=0, \forall x \in S$ est une condition nécessaire et suffisante pour que le spectre de $H$ dans $A$ soit purement continu.

Pour cela, considérons le vecteur $E_{H}(\{A\}) \delta_{x}$, et notons $v$ la mesure spectrale de $H$ prise entre ce vecteur à droite et à gauche. D'après le théorème de Wiener, la mesure de la diagonale de $\mathbb{R} \times \mathbb{R}$ par la mesure $v \otimes v$ est nulle si et seulement si $v$ est continue. C'est-à-dire que $E_{H}(A) \delta_{x} \in \mathscr{H}_{c}(H), \mathscr{H}_{c}(H)$ étant le sous espace de continuité de $\mathscr{H}={ }^{\ell 2}(S)$ associé à $H$, si et seulement si $R_{H}(x, x ; A)=0$. Le fait que $\mathscr{H}_{c}(H)$ soit un fermé achève la preuve.

Corollaire IV.7 Soit $\mu$ une mesure de probabilité sur $\mathfrak{S}(S)$.

a) $S i \exists x \in S, \lim _{\Lambda \uparrow S} R_{\mu}^{\Lambda}(x, x ; A)>0$ alors l'opérateur $H$ possède du spectre purement ponctuel dans $A$ avec une $\mu$-probabilité non nulle.

b) Une condition nécessaire et suffisante pour que le spectre de $H$ dans $A$ soit purement continu pour $\mu$-presque tout $H$ est que

$$
\forall x \in S, R_{\mu}(x, x ; A)=\lim _{\varepsilon \rightarrow 0} \lim _{\Lambda \uparrow S} R_{\mu}^{\Lambda}(x, y ; A, \varepsilon)=0 .
$$

Preuve. Comme les quantités $R_{H}$ et $R_{H}^{A}$ sont positives et bornées par 1 , on peut permuter la moyenne en $\mu$ et les diverses limites. Les propositions a) et b) du corollaire ci-dessus sont alors des conséquences immédiates des propositions correspondantes du Théorème IV.6.

Remarque. La quantité $R_{\mu}^{A}(x, x ; A)$ a été utilisée dans la littérature physique ${ }^{11}$ pour, en particulier dans des études numériques, distinguer effectivement les états localisés des états délocalisés. Notons cependant que $\lim _{\Lambda \uparrow S} R_{\mu}^{\Lambda}(x, x ; A)=0$ n'implique pas nécessairement que le spectre soit purement continu dans $A$, car seule l'inégalité $\lim _{\Lambda \uparrow S} R_{\mu}^{\Lambda}(x, x ; A) \leqq R_{\mu}(x, x ; A)$ est toujours vraie et il existe des contre-exemples simples dans lesquels le membre de gauche est nul et pas celui de droite.

En l'absence de justification supplémentaire, $\lim _{\Lambda \uparrow S} R_{\mu}^{\Lambda}(x, x ; A)=0$ ne peut être considéré comme un critère de la présence d'états délocalisés, mais peut seulement indiquer des bornes à la transition d'Anderson ou au seuil de mobilité. Pour travailler avec certitude il est préférable d'utiliser le critère $\lim _{\varepsilon \rightarrow 0} \lim _{\Lambda \uparrow S} R_{\mu}^{\Lambda}(x, x ; A, \varepsilon)=0$.

\section{Sur la conductivité et la constante de diffusion}

Nous allons maintenant discuter d'un aspect plus proprement physique de notre problème, soit de la conductivité électrique et de la constante de diffusion. Pour cela nous interprétons l'opérateur $H$ comme l'hamiltonien à une particule d'un

11 En fait, $1 / R_{H}(x, x ; A)$ y est souvent appelé rapport de participation 
système d'électrons indépendants soumis à l'influence d'un potentiel aléatoire qui pourrait être créé par des impuretés, c'est-à-dire que $H$ est le générateur de l'évolution de la fonction d'onde de l'électron par le semi-groupe $\exp \{-i t H\}$. Il est alors d'un grand intérêt physique de savoir si un tel système se comporte comme un isolant ou un conducteur. Dans ce but on étudie généralement la conductivité électrique statique et la constante de diffusion du système. On s'attend à ce que toutes deux soient nulles lorsque la température tend vers 0 , si le système est un isolant. La définition de ces grandeurs dans un cadre plus général que celui de la réponse linéaire pose de nombreux problèmes, qui ne sont pas vraiment résolus à l'heure actuelle. On pense en général que des modèles comme ceux que nous considérons ici, ne peuvent fournir une définition physiquement acceptable de la conductivité que dans le cadre d'une telle théorie. Pour poser le problème dans un cadre physiquement correct, indépendamment de la théorie de la réponse linéaire, il faudrait modifier le modèle, en y ajoutant par exemple une interaction des électrons avec des phonons. Aussi prendrons nous comme définition de la conductivité électrique celle qui est donnée par la formule de Greenwood-Kubo. Nous allons montrer que, dans ce cadre, si tous les états sont par exemple exponentiellement localisés, la conductivité statique est toujours nulle à température non nulle, et que la constante de diffusion s'annule également. Le système sera alors un isolant. Nous montrerons encore que cette conductivité statique s'annule quand la température tend vers 0 lorsque le niveau de Fermi est situé dans la région d'énergie correspondant aux états localisés.

Supposons maintenant que $S$ soit un ensemble de points de $\mathbb{R}^{d}$. Supposons que le champ électrique soit appliqué dans une direction $\vec{a}$, l'opérateur de position associé à cette direction sera noté $X_{1}$, et les composantes selon $\vec{a}$ des positions des points de $S$ seront indexées par 1: $x_{1}, y_{1} \ldots$. L'opérateur $X_{1}$ est un opérateur autoadjoint qui admet $f(S)$ pour cœur. La partie diagonale du tenseur de conductivité, correspondant à la direction choisie $\vec{a}$, est donnée pour une boîte finie $A$ de $S$ par l'expression suivante en théorie de la réponse linéaire

$$
\begin{aligned}
\operatorname{Re} \sigma_{H}^{\Lambda}(\omega)= & \frac{\pi e^{2}}{|\Lambda|} \omega \sum_{x, y \in \Lambda} x_{1} \cdot y_{1} \sum_{\lambda, \lambda^{\prime} \in \operatorname{Sp} H_{A}} \\
& \times\left\langle\delta_{x}, E^{\Lambda}(\{\lambda\}) \delta_{y}\right\rangle\left\langle\delta_{y}, E^{\Lambda}\left(\left\{\lambda^{\prime}\right\}\right) \delta_{x}\right\rangle\left(f(\lambda)-f\left(\lambda^{\prime}\right)\right) \delta_{\lambda+\hbar \omega}\left(\lambda^{\prime}\right)
\end{aligned}
$$

$\omega \geqq 0$ désigne la fréquence, $\hbar$ est la constante de Planck, e la charge de l'électron, $\beta$ la température inverse et $f(\lambda)$ la distribution de Fermi-Dirac

$$
f(\lambda)=\{\exp \beta(\lambda-\mu)+1\}^{-1}
$$

$\mu$ étant le potentiel chimique.

Lorsque les vecteurs propres associés à $H$ ne sont pas dégénérés, cette formule peut se réécrire sous la forme

$$
\begin{aligned}
\operatorname{Re} \sigma_{H}^{A}(\omega)= & \frac{\pi e^{2}}{\hbar} \omega \sum_{\substack{\lambda, \lambda^{\prime} \in \operatorname{Sp} H_{A} \\
\lambda \neq \lambda^{\prime}}}\left\{X_{1}\left(\lambda, \lambda^{\prime}\right)\right\}^{2} \\
& \times\left\{f\left(\lambda^{\prime}\right)-f(\lambda)\right\} \delta_{\lambda+\hbar \omega}\left(\lambda^{\prime}\right)
\end{aligned}
$$


où

$$
X_{1}\left(\lambda, \lambda^{\prime}\right)=\sum_{x \in \Lambda} x_{1} \psi_{\lambda}(x) \psi_{\lambda^{\prime}}(x)
$$

représente l'élément de matrice de l'opérateur de position dans la direction $X_{1}$ dans la base formée par les vecteurs propres $\psi_{\lambda}$ de l'hamiltonien $H^{\Lambda}$. C'est sous cette forme que se trouve exprimé $\operatorname{Re} \sigma_{H}^{\Lambda}(\omega)$ chez Thouless [30] par exemple.

On ne s'attend pas nécessairement (notamment pour des distributions de probabilité singulières) à ce que la limite thermodynamique d'une telle quantité existe, autrement qu'au sens de la convergence faible d'une mesure pour des suites de boites appropriées. Aussi allons nous introduire, comme dans le cas de la densité d'états, une grandeur d'accès mathématique plus aisé, mais qui contient la même information physique, soit la conductivité intégrée.

Définitions. La conductivité intégrée associée à l'opérateur $H$, dans la boite finie $\Lambda$, pour la fréquence $\omega$ est définie par

$$
\operatorname{Re} \bar{\sigma}_{H}^{A}(\omega)=\int_{0}^{\omega} \operatorname{Re} \sigma_{H}^{A}\left(\omega^{\prime}\right) d \omega^{\prime}
$$

soit au vu de l'expression (V.1)

$$
\begin{aligned}
\operatorname{Re} \bar{\sigma}_{H}^{\Lambda}(\omega)= & \frac{\pi e^{2}}{\hbar|\Lambda|} \sum_{x, y \in \Lambda} x_{1} \cdot y_{1} \sum_{\substack{\lambda, \lambda^{\prime} \in \operatorname{Sp} H_{\Lambda} \\
\hbar \omega \geqq \lambda^{\prime}-\lambda \geqq 0}} \\
& \times\left(\lambda^{\prime}-\lambda\right)\left(f(\lambda)-f\left(\lambda^{\prime}\right)\right)\left\langle\delta_{x}, E_{H}^{\Lambda}(\{\lambda\}) \delta_{y}\right\rangle \times\left\langle\delta_{y}, E_{H}^{\Lambda}\left(\left\{\lambda^{\prime}\right\}\right) \delta_{x}\right\rangle
\end{aligned}
$$

La conductivité intégrée associée à l'opérateur $H$, pour la fréquence $\omega$ est alors définie $^{12}$ par

$$
\bar{\sigma}_{H}(\omega)=\liminf _{\Lambda \uparrow S} \bar{\sigma}_{H}^{\Lambda}(\omega)
$$

et la conductivité statique $\sigma_{H}$ associée à $H$ par

$$
\sigma_{H}=\liminf _{\Lambda \uparrow S} \frac{1}{\omega} \bar{\sigma}_{H}^{\Lambda}(\omega)
$$

Nous pouvons alors voir facilement que pour une très large classe d'opérateurs $H$, la conductivité intégrée est bornée indépendamment de la fréquence:

Proposition V.1. Soit un opérateur $H, H \in \mathfrak{H}$. Supposons que

Alors pour tout $\Lambda$ et $\omega$

$$
K(H)=\operatorname{Sup}_{x \in S} \sum_{y \in S}\left(x_{1}-y_{1}\right)^{2}|H(x, y)|<\infty .
$$

$$
\operatorname{Re} \bar{\sigma}_{H}^{\Lambda}(\omega) \leqq \operatorname{Re} \bar{\sigma}_{H}^{\Lambda}(\infty) \leqq \frac{\pi e^{2}}{2 \hbar} K(H)
$$

12 Remarque. La conductivité peut a priori dépendre de la façon dont la boîte $\Lambda$ croit vers $S$. Il est physiquement sous entendu dans cette section que la boîte croit macroscopiquement dans toutes les directions, typiquement un cube ou une sphère 
Preuve. Nous réécrivons d'abord $\operatorname{Re} \bar{\sigma}_{H}^{A}(\omega)$ sous la forme

$$
\begin{aligned}
\operatorname{Re} \bar{\sigma}_{H}^{A}(\omega)= & \frac{\pi e^{2}}{\hbar|\Lambda|} \sum_{\substack{\lambda, \lambda^{\prime} \in \operatorname{Sp} H_{A} \\
\hbar \omega \geqq \lambda^{\prime}-\lambda \geqq 0}}\left(\lambda^{\prime}-\lambda\right)\left(f(\lambda)-f\left(\lambda^{\prime}\right)\right) \\
& \cdot \operatorname{Tr}\left(X_{1} E^{\Lambda}(\{\lambda\}) X_{1} E^{\Lambda}\left(\left\{\lambda^{\prime}\right\}\right)\right)
\end{aligned}
$$

et donc puisque $\left(\lambda^{\prime}-\lambda\right)\left(f(\lambda)-f\left(\lambda^{\prime}\right)\right) \geqq 0$ et

$$
\begin{gathered}
\operatorname{tr}\left(X_{1} E^{\Lambda}(\{\lambda\}) X_{1} E^{\Lambda}\left(\left\{\lambda^{\prime}\right\}\right)\right) \\
=\operatorname{tr}\left(E^{\Lambda}(\{\lambda\}) X_{1} E^{\Lambda}(\{\lambda\}) \times E^{\Lambda}(\{\lambda\}) X_{1} E^{\Lambda}\left(\left\{\lambda^{\prime}\right\}\right)\right) \geqq 0
\end{gathered}
$$

nous voyons que

$$
\operatorname{Re} \bar{\sigma}_{H}^{A}(\omega) \leqq \operatorname{Re} \bar{\sigma}_{H}^{A}(\infty)
$$

Par ailleurs on voit facilement que

$$
\begin{aligned}
\operatorname{Re} \bar{\sigma}_{H}^{\Lambda}(\infty)=\frac{\pi e^{2}}{\hbar|\Lambda|} \frac{1}{2} \sum_{\lambda, \lambda^{\prime} \in \operatorname{Sp} H_{\Lambda}} & \left(\lambda^{\prime}-\lambda\right)\left(f(\lambda)-f\left(\lambda^{\prime}\right)\right) \operatorname{tr}\left(X_{1} E^{\Lambda}(\{\lambda\}) X_{1} E^{\Lambda}\left(\left\{\lambda^{\prime}\right\}\right)\right) \\
= & -\frac{\pi e^{2}}{\hbar|\Lambda|} \sum_{\lambda \in \operatorname{Sp} H_{\Lambda}} \lambda f(\lambda) \operatorname{tr}\left(X_{1}^{2} E^{\Lambda}(\{\lambda\})\right) \\
& +\sum_{\lambda, \lambda^{\prime} \in \operatorname{Sp} H_{A}} \lambda f\left(\lambda^{\prime}\right) \operatorname{tr}\left(X_{1} E^{\Lambda}(\{\lambda\}) X_{1} E^{\Lambda}\left(\left\{\lambda^{\prime}\right\}\right)\right)
\end{aligned}
$$

c'est-à-dire

$$
\operatorname{Re} \bar{\sigma}_{H}^{\Lambda}(\infty)=\frac{\pi e^{2}}{\hbar|\Lambda|} \operatorname{tr}\left(X_{1}\left[H, X_{1}\right] f(H)\right)
$$

On déduit facilement de (V.11) en utilisant que $0 \leqq f(\lambda) \leqq 1$

$$
\operatorname{Re} \bar{\sigma}_{H}^{\Lambda}(\omega) \leqq \frac{\pi e^{2}}{2 \hbar} \frac{1}{\Lambda} \cdot \sum_{x, y \in \Lambda}\left(x_{1}-y_{1}\right)^{2}|H(x, y)|
$$

Nous allons maintenant obtenir un critère simple, assurant que la conductivité statique est nulle si tous les états sont suffisamment localisés.

Théorème V.2. Soit $H \in \mathfrak{H}$. Supposons que $H$ satisfasse l'une des deux conditions suivantes
a) $\Delta_{X}=\liminf _{\Lambda \uparrow S} \frac{1}{|\Lambda|} \sum_{x, y \in \Lambda}\left(x_{1}-y_{1}\right)^{2} R_{H}^{\Lambda}(x, y ; \mathbb{R})<\infty$,
b) $m=\liminf _{\Lambda \uparrow S} \frac{1}{|\Lambda|} \sum_{x, y \in \Lambda}\left(\mathrm{x}_{1}-\mathrm{y}_{1}\right)^{2}\left(\bar{\varrho}_{H}^{\Lambda}(\mathrm{x}, \mathrm{y} ; \mathbb{R})\right)^{2}<\infty$.

Alors la conductivité intégrée satisfait l'inégalité

$$
\operatorname{Re} \bar{\sigma}_{H}(\omega) \leqq \beta \omega^{2} \frac{\pi e^{2}}{2} \Delta_{X} \leqq \beta \omega^{2} \frac{\pi e^{2}}{2} m
$$

et la conductivité statique est nulle. 
Preuve. Utilisant que $\operatorname{Tr}\left(X_{1} E^{\Lambda}(\{\lambda\}) X_{1} E^{\Lambda}\left(\left\{\lambda^{\prime}\right\}\right)\right) \geqq 0$ et que $\left|\left(\lambda^{\prime}-\lambda\right)\left(f(\lambda)-f\left(\lambda^{\prime}\right)\right)\right|$ $\leqq \beta \hbar \omega^{2}$ si $\hbar \omega \geqq \lambda^{\prime}-\lambda \geqq 0$, nous pouvons majorer la conductivité intégrée par

$$
\begin{aligned}
& \text { Mais } \operatorname{Re} \bar{\sigma}_{H}^{\Lambda}(\omega) \leqq \beta \omega^{2} \frac{\pi e^{2}}{|\Lambda|} \sum_{\substack{\lambda, \lambda^{\prime} \in \operatorname{Sp} H^{\Lambda} \\
\lambda \neq \lambda^{\prime}}} \operatorname{Tr}\left(X_{1} E^{\Lambda}(\{\lambda\}) X_{1} E^{\Lambda}\left(\left\{\lambda^{\prime}\right\}\right)\right) . \\
& \sum_{\substack{\lambda, \lambda^{\prime} \in \operatorname{Sp} H^{\prime} \\
\lambda^{\prime} \neq \lambda}} \operatorname{Tr}\left(X_{1} E^{\Lambda}(\{\lambda\}) X_{1} E^{\Lambda}\left(\left\{\lambda^{\prime}\right\}\right)\right) \\
& =\frac{1}{2} \sum_{\substack{\lambda, \lambda^{\prime} \in \operatorname{Sp} H^{\prime} \\
\lambda^{\prime} \neq \lambda}} \sum_{x, y \in \Lambda}\left\langle\delta_{x}, E^{\Lambda}(\{\lambda\}) \delta_{y}\right\rangle\left\langle\delta_{y}, E^{\Lambda}\left(\left\{\lambda^{\prime}\right\}\right) \delta_{x}\right\rangle \cdot\left\{-\left(x_{1}-y_{1}\right)^{2}+x_{1}^{2}+y_{1}^{2}\right\} \\
& =\frac{1}{2} \sum_{x, y \in \Lambda}\left(x_{1}-y_{1}\right)^{2} \sum_{\lambda \in \operatorname{Sp} H^{\Lambda}}\left\langle\delta_{x}, E^{\Lambda}(\{\lambda\}) \delta_{y}\right\rangle\left\langle\delta_{y}, E^{\Lambda}(\{\lambda\}) \delta_{x}\right\rangle \\
& =\frac{1}{2} \sum_{x, y \in \Lambda}\left(x_{1}-y_{1}\right)^{2} R_{H}^{\Lambda}(x, y ; \mathbb{R})
\end{aligned}
$$

ce qui assure la première inégalité de (V.14) en passant à la limite $\Lambda \uparrow S$. La seconde s'obtient en notant que

$$
R_{H}^{\Lambda}(x, y ; \mathbb{R}) \leqq\left(\bar{\varrho}_{H}^{\Lambda}(x, y ; \mathbb{R})\right)^{2}
$$

d'où $\Delta_{X} \leqq m$. L'annulation de la conductivité statique découle directement de ces bornes.

Remarque. Les inégalités (V.14) sont toujours vraies, même si $\Delta_{x}$ et $m$ sont infinis. La notation $\Delta_{X}$ indique que la quantité considérée est un écart de position; l'écart de position associé à l'opérateur $\mathfrak{H}$ dans la boîte $\Lambda$ peut être défini comme

$$
\Delta_{X}^{\Lambda}(H)=\frac{1}{|\Lambda|} \operatorname{Tr}\left\{\int_{\mathbb{R} \times \mathbb{R} \backslash \Delta} X_{1} E_{H}^{\Lambda}(d \lambda) X_{1} E_{H}^{\Lambda}\left(d \lambda^{\prime}\right)\right\}
$$

où $\Delta$ désigne la diagonale de $\mathbb{R} \times \mathbb{R}$. Si $H^{\Lambda}$ n'a pas de valeur propre dégénérée l'écart de position s'écrit encore

$$
\Delta_{X}^{\Lambda}(H)=\frac{1}{|\Lambda|} \sum_{\lambda \in \operatorname{Sp} H^{\Lambda}}\left\{\left\langle\psi_{\lambda}, X_{1}^{2} \psi_{\lambda}\right\rangle-\left(\left\langle\psi_{\lambda}, X_{1} \psi_{\lambda}\right\rangle\right)^{2}\right\}
$$

Il est souvent plus facile de travailler avec des critères portant sur des valeurs moyennes. Aussi donnerons nous le critère utile suivant:

Corollaire V.3. Soit $H \in \mathfrak{H}$. Si $\mu$ est une mesure de probabilité sur $\mathfrak{H}$ satisfaisant l'une des conditions suivantes

a) $\liminf _{\Lambda \uparrow S} \frac{1}{|\Lambda|} \sum_{x, y \in \Lambda}\left(x_{1}-y_{1}\right)^{2} R_{\mu}^{\Lambda}(x, y ; \mathbb{R})<\infty$,
b) $\liminf _{\Lambda \uparrow S} \frac{1}{|\Lambda|} \sum_{x, y \in \Lambda}\left(x_{1}-y_{1}\right)^{2} \bar{\varrho}_{\mu}^{\Lambda}(x, y ; \mathbb{R})<\infty$.

Alors $\mu$-presque tout opérateur $H$ a une conductivité statique nulle et une conductivité intégrée satisfaisant $\operatorname{Re} \bar{\sigma}_{H}(\omega)=0\left(\omega^{2}\right)$.

Preuve. Ce résultat est une conséquence simple du théorème précédent, en utilisant le lemme de Fatou. 
Remarque. L'idée de relier la conductivité à ce que nous avons appelé $\Delta_{X}$, soit l'écart de position moyen dans les états propres, est apparue initialement chez Halperin [12]. Ce lien a été plus ou moins formalisé par divers auteurs, dont Ishii [13]. Il semble par contre que la connexion avec les fonctions de correlation (partie $b$ ) apparaisse ici pour la première fois. C'est d'ailleurs la forme $b$ ) du corollaire cidessus que nous utiliserons pour montrer qu'en dimension un, la conductivité statique s'annule avec probabilité un, pour une large classe d'équations de Schrödinger discrétisées.

Le Théorème V.2 et son Corollaire V.3 expriment que si tous les états sont localisés en un sens approprié, la conductivité électrique statique est nulle pour toute température, indépendamment de la position du niveau de Fermi. Ce sera le cas pour l'opérateur de Schrödinger discrétisé unidimensionnel (voir Sect. IX). On désire cependant un résultat plus général, d'intérêt lorsqu'il existe des états étendus, à savoir que si l'énergie de Fermi $\mu$ appartient à la composante purement ponctuelle du spectre, c'est-à-dire à la région d'energies correspondant aux états localisés (ou a fortiori si $\mu$ est en dehors du spectre) alors la conductivité électrique statique s'annule lorsque la température tend vers zéro. Le théorème suivant exprime un tel résultat.

Théorème V.4. Soit $H \in \mathfrak{H}$, tel que

$$
K(H)=\operatorname{Sup}_{x \in S} \sum_{y \in S}\left(x_{1}-y_{1}\right)^{2}|H(x, y)|<\infty
$$

et supposons que l'une des conditions suivantes soient satisfaites

(a) $\Delta_{X}([\mu-\alpha, \mu+\alpha])$

$$
=\liminf _{\Lambda \uparrow S} \frac{1}{|\Lambda|} \sum_{x, y \in \Lambda}\left(x_{1}-y_{1}\right)^{2} R_{H}^{\Lambda}(x, y ;[\mu-\alpha, \mu+\alpha])<\infty
$$

(b) $m([\mu-\alpha, \mu+\alpha])$

$$
=\liminf _{\Lambda \uparrow S} \frac{1}{|\Lambda|} \sum_{x, y \in \Lambda}\left(x_{1}-y_{1}\right)^{2} \bar{\varrho}_{H}^{\Lambda}(x, y ;[\mu-\alpha, \mu+\alpha])<\infty
$$

où $\mu$ désigne l'énergie de Fermi dans (V.2).

Alors pour $\hbar \omega<\alpha / 2$ la conductivité intégrée satisfait l'inégalité

$$
\begin{aligned}
\operatorname{Re} \bar{\sigma}_{H}(\omega) & \leqq \beta \omega^{2} \frac{\pi e^{2}}{2} \Delta_{X}([\mu-\alpha, \mu+\alpha])+2 \pi \frac{e^{2}}{\hbar} K(H) e^{-\beta \alpha / 4} \\
& \leqq \beta \omega^{2} \frac{\pi e^{2}}{2} m([\mu-\alpha, \mu+\alpha])+2 \pi \frac{e^{2}}{\hbar} K(H) e^{-\beta \alpha / 4}
\end{aligned}
$$

et en particulier la conductivité électrique statique tend vers zéro quand la température tend vers zéro.

Preuve. Considérons la formule (V.10) exprimant la conductivité intégrée. Nous y séparons la sommation sur $\lambda$ en trois parties correspondant respectivement à $\lambda \in[\mu-\alpha, \mu+\alpha], \lambda>\mu+\alpha$ et $\lambda<\mu-\alpha$. Nous traitons la première de ces régions de façon analogue à (V.15) (V.16), mais en y conservant la contrainte $\lambda \in[\mu-\alpha, \mu+\alpha]$ ce qui conduit au premier terme dans la borne sur $\operatorname{Re} \bar{\sigma}_{H}(\omega)$. La seconde partie, 
$\lambda>\mu+\alpha$ se traite de façon parallèle à la preuve de (V.9), mais en utilisant dans (V.10) la majoration

$$
\begin{aligned}
f(\lambda)-f\left(\lambda^{\prime}\right) & \leqq\left(\sqrt{f(\lambda)}+\sqrt{f\left(\lambda^{\prime}\right)}\right)\left(\sqrt{f(\lambda)}-\sqrt{f\left(\lambda^{\prime}\right)}\right) \\
& \leqq 2 e^{-\beta \alpha / 2}\left(\sqrt{f(\lambda)}-\sqrt{f\left(\lambda^{\prime}\right)}\right) .
\end{aligned}
$$

Enfin la dernière partie $\lambda<\mu-\alpha$ se traite de façon semblable mais en utilisant que $\lambda^{\prime}-\lambda \leqq \hbar \omega<\alpha / 2$ et dans (V.10) la majoration

$$
\begin{aligned}
f(\lambda)-f\left(\lambda^{\prime}\right) & =\left(1-f\left(\lambda^{\prime}\right)\right)-(1-f(\lambda)) \\
& \leqq\left(\sqrt{1-f\left(\lambda^{\prime}\right)}+\sqrt{1-f(\lambda))}\left(\sqrt{1-f\left(\lambda^{\prime}\right)}-\sqrt{1-f(\lambda)}\right)\right. \\
& \leqq 2 e^{-\beta \alpha / 4}\left(\sqrt{1-f\left(\lambda^{\prime}\right)}-\sqrt{1-f(\lambda)}\right) .
\end{aligned}
$$

Il est clair que si $\mu$ est une mesure de probabilité sur $\mathfrak{S}$, telle que $K(H)<\infty$ pour $\mu$-presque tout $H$ et si $R_{\mu}^{\Lambda}(x, y ;[\mu-\alpha, \mu+\alpha])$ ou $\varrho_{\mu}^{\Lambda}(x, y ;[\mu-\alpha, \mu+\alpha])$ satisfont des propriétés analogues à (V.20) et (V.21) les conclusions du Théorème V.4 s'appliqueront à $\mu$-presque tout $H$. Nous n'expliciterons pas le corollaire correspondant.

Une autre grandeur d'intérêt physique est la constante de diffusion, proportionnelle à la mobilité. Elle peut se définir de la manière suivante:

Définition. Soit $H \in \mathfrak{H}$. La constante de diffusion d'une particule d'énergie comprise dans un intervalle $A$, située initialement au point $y$ est donnée par

$$
D_{H}(y ; A)=\lim _{\varepsilon \rightarrow 0} \varepsilon^{2} \sum_{x \in S}(x-y)^{2} \int_{0}^{\infty} e^{-\varepsilon t}\left|\left\langle\delta_{x}, e^{i t H} E(A) \delta_{y}\right\rangle\right|^{2} d t
$$

et la constante de diffusion associée à l'hamiltonien $H$ pour l'intervalle $A$ est définie $\operatorname{par} D_{H}(A)=\operatorname{Sup} D_{H}(y ; A)$.

La constante de diffusion moyenne pour une probabilité $\mu$ sur $\mathfrak{H}$ est donnée par:

$$
D_{\mu}(A)=\sup _{y \in S} \mu\left(D_{H}(y ; A)\right) .
$$

C'est cette définition que l'on trouve dans Thouless [30] par exemple.

Nous avons alors le critère suivant:

Théorème V.5. Soit $H \in \mathfrak{H}$, tel que

$$
\sum_{x \in S}(x-y)^{2}\left(\bar{\varrho}_{H}(x, y ; A)\right)^{2}<\infty .
$$

Alors la constante de diffusion $D_{H}(y ; A)$ est nulle.

Preuve. Le résultat se déduit de la remarque

$$
\begin{aligned}
& \sum_{x \in S}(x-y)^{2} \int_{0}^{\infty} e^{-\varepsilon t}\left|\left\langle\delta_{x}, e^{i t H} E(A) \delta_{v}\right\rangle\right|^{2} d t \\
& \leqq \sum_{x \in S}(x-y)^{2} \varepsilon^{-1}\left(\bar{\varrho}_{H}(x, y ; A)\right)^{2} . \square
\end{aligned}
$$

Un critère correspondant pour les valeurs moyennes est donné par le 
Corollaire V.6. Soit $\mu$ une mesure de probabilité sur $\mathfrak{H}$, satisfaisant

$$
\sum_{x \in S}(x-y)^{2} \bar{\varrho}_{\mu}(x, y ; A)<\infty .
$$

Alors la constante de diffusion $D_{H}(y ; A)$ est nulle pour $\mu$-presque tout $H$.

\section{Un formalisme général}

Dans les sections précédentes, nous avons établi des critères permettant d'assurer que sous certaines conditions, presque tout opérateur $H$ a un spectre purement ponctuel, ou purement continu, ou encore que la conductivité électrique statique est nulle. L'utilisation de ces critères nécessite de calculer des quantités de la forme

ou

$$
\sum_{\lambda \in \mathrm{Sp} H^{\Lambda} \cap A}\left|\psi_{\lambda, H}^{\Lambda}(x) \psi_{\lambda, H}^{A}(y)\right|
$$

$$
\sum_{\lambda \in \operatorname{Sp} H^{\Lambda} \cap A}\left[\psi_{\lambda, H}^{\Lambda}(x)\right]^{4} .
$$

D’autres quantités d'intérêt se mettent sous une forme semblable; ainsi la «densité d'état» est obtenue par limite de $\frac{1}{\Lambda} \sum_{\lambda \in \mathrm{Sp} H^{\Lambda} \cap A} 1$; elles sont baptisées par les physiciens «quantités à une énergie» parce que ne faisant intervenir qu'une seule valeur propre de $H^{A}$ et la fonction propre associée.

Nous souhaitons donc calculer des quantités de ce type, mais en général il est difficile de diagonaliser $H^{\Lambda}$ et de calculer ses valeurs propres et vecteurs propres. Nous développons ici une approche alternative, applicable dès que la mesure $\mu$ sur $\mathfrak{H}(S)$ est absolument continue sur les éléments diagonaux - le potentiel - pour une suite de boîtes $\Lambda$ croissant vers $S$. Le but de cette section est d'obtenir dans ce cas une formule explicite permettant de calculer les observables du système dans un volume fini à l'aide d'intégrations, de même que la formule de Gibbs permet un tel calcul en Mécanique Statistique.

L'idée est la suivante. Fixons $H_{N D}^{\Lambda}$; s'il est difficile de calculer valeurs propres et vecteurs propres de $H^{\Lambda}=H_{N D}^{\Lambda}+H_{D}^{\Lambda}$, au contraire, étant donné $\lambda$ et $\psi$ on calcule facilement les termes diagonaux $H_{D}^{\Lambda}$ tels que $\lambda$ et $\psi$ soient valeur propre et vecteur propre de $H^{\Lambda}$ pour $H_{N D}^{\Lambda}$ fixe. D'où l'idée d'utiliser cette application $(\lambda, \psi) \rightarrow H_{D}^{\Lambda}$ pour transporter la mesure sur $H_{D}, \mu\left(H_{D} \mid H_{N D}\right)$, en une mesure sur $(\lambda, \psi)$ déterminant la densité de probabilité pour que $\lambda, \psi$ soit un couple valeur propre, vecteur propre de $H^{\Lambda}$.

Soulignons qu'une telle approche avait été initiée d'une manière non rigoureuse par Eggarter [8]: cependant le bon changement de variable n'avait pu être trouvé dans ce travail ce qui y restreignait l'approche au cas de la dimension un, et même dans ce cas, les résultats étaient trop compliqués pour être exploités.

Simplifions ou précisons tout d'abord les notations: $H_{N D}^{A}$ sera conservé fixe dans les Théorèmes VI.1 et VI.2. Nous y réappelons la partie diagonale $H_{D}^{\Lambda}$ : $\omega=\left\{\omega_{x}\right\}_{x \in A}=\{H(x, x)\}_{x \in A}$. La mesure $d \mu\left(H_{D}^{A} \mid H_{N D}^{\Lambda}\right)$ sera supposée absolument continue:

$$
d \mu\left(H_{D}^{\Lambda} \mid H_{N D}^{\Lambda}\right)=v_{H_{N D}}(\omega) d \omega .
$$


Si $\lambda$ est valeur propre de $H^{\Lambda}=H_{N D}^{\Lambda}+\omega, \pm \frac{\psi_{\lambda}}{\left\|\psi_{\lambda}\right\|}, \psi_{\lambda}=\left\{\psi_{\lambda}(x)\right\}_{x \in \Lambda}$, dénotera les deux orientations de son vecteur propre normalisé. Il sera naturel de travailler aussi dans l'espace projectif $\mathbb{P}^{|\Lambda|-1}$; nous y utiliserons la carte obtenue par projection stéréographique de base un point donné de $\Lambda$, disons 0 :

$$
\alpha(x)=\frac{\psi(x)}{\psi(0)}, \alpha(0)=1, \alpha=\left\{\alpha_{x}\right\}_{x \in \Lambda}
$$

et nous noterons

$$
\|\alpha\|^{2}=\sum_{x \in \Lambda} \alpha(x)^{2}=1+\sum_{x \in \Lambda \backslash 0} \alpha(x)^{2} .
$$

Introduisons maintenant les observables que nous voulons calculer:

Définition. Soit $H^{\Lambda}=H_{N D}^{\Lambda}+\omega$. Les «observables à une énergie» sont définies par la formule suivante, lorsque $H$ n'a pas de valeur propre dégénérée ${ }^{13}$ :

$$
\begin{aligned}
f_{g}^{\Lambda}(\omega) & =\sum_{\lambda \in \mathrm{Sp} H^{\Lambda}}\left\{g\left(\lambda, \frac{\psi_{\lambda}}{\left\|\psi_{\lambda}\right\|}\right)+g\left(\lambda,-\frac{\psi_{\lambda}}{\left\|\psi_{\lambda}\right\|}\right)\right\} \\
& =\sum_{\lambda \in \mathrm{Sp} H^{\Lambda}}\left\{g\left(\lambda, \frac{\alpha_{\lambda}}{\left\|\alpha_{\lambda}\right\|}\right)+g\left(\lambda,-\frac{\alpha_{\lambda}}{\left\|\alpha_{\lambda}\right\|}\right)\right\}
\end{aligned}
$$

où $g(\lambda, \psi)$ est une fonction mesurable de $\lambda, \psi$.

Exemples. Soit $\chi_{A}(\lambda)$ la fonction caractéristique de l'intervalle $A$. Alors le choix $g(\lambda, \psi)=\frac{1}{2|\Lambda|} \chi_{A}(\lambda)$ conduit à une observable $f_{g}^{\Lambda}(\omega)$ qui est la «densité d'état intégrée dans l'intervalle $A »$, à volume fini $\Lambda$; le choix $g(\lambda, \psi)=\frac{1}{2} \chi_{A}(\lambda) \frac{\left|\psi_{(x)} \psi_{(y)}\right|}{\|\psi\|^{2}}$ conduit à $f_{g}^{\Lambda}(\omega)=\varrho^{\Lambda}(x, y ; A)$, la «corrélation de $H$ dans $A$ entre les points $x$ et $y$ », corrélation qui apparaît dans les critères des Théorèmes IV.4 et V.2 sur le spectre purement ponctuel de $H$ ou l'absence de conductivité statique; le choix $g(\lambda, \psi)$ $=\frac{1}{2} \chi_{A}(\lambda)\left(\frac{\psi(x)}{\|\psi\|}\right)^{4}$ conduit à $f_{g}^{\Lambda}(\omega)=R_{H_{N D}+\omega}^{\Lambda}(x, x ; A)$ la «mesure de récurrence au point $x$ de $H$ » qui apparaît dans l'étude du spectre purement continu de $H$ (critère du Théorème IV.6).

Ce que nous voulons calculer, c'est $\varlimsup_{\frac{\lim }{\Lambda \uparrow S}} \mu\left(f^{\Lambda}\right)$. Mais

$$
\mu\left(f^{\Lambda}\right)=\int \mu\left(f^{\Lambda} \mid H_{N D}^{\Lambda}\right) d \mu\left(H_{N D}^{\Lambda}\right)
$$

et nous sommes conduits à calculer $\mu\left(f^{\Lambda} \mid H_{N D}^{\Lambda}\right)$. Puisque la mesure $\mu\left(\cdot \mid H_{N D}^{\Lambda}\right)$ est supposée absolument continue dans cette section, nous calculerons:

$$
v_{H_{N D}}\left(f^{\Lambda}\right)=\int f_{g}^{\Lambda}(\omega) v_{H_{N D}}(\omega) d \omega .
$$

Considérons maintenant l'équation aux valeurs propres:

$$
H^{\Lambda} \psi=\lambda \psi
$$

13 La mesure $\mu\left(\cdot \mid H_{N D}^{A}\right)$ étant supposée absolument continue dans cette section, en vue de la Proposition II.1, il n'est pas nécessaire de définir $f_{g}^{\Lambda}(\omega)$ lorsque $H^{\Lambda}$ a des valeurs propres dégénérées 
soit :

$$
\forall x \in \Lambda, \sum_{z \in \Lambda, z \neq x} H(x, z) \psi(z)+\omega_{x} \psi(x)=\lambda \psi(x)
$$

ou encore lorsque $\psi(0) \neq 0$

$$
\forall x \in \Lambda, \sum_{z \in \Lambda, z \neq x} H(x, z) \alpha(z)+\omega_{x} \alpha(x)=\lambda \alpha(x) .
$$

Introduisons alors les deux applications $\omega$ et $h$ suivantes, de $\mathbb{R}^{A}$ dans $\mathbb{R}^{A}$ ou $\mathbb{R}^{\Lambda+1}$ dans $\mathbb{R}^{\Lambda+1}$, définies lorsque $\alpha(x) \neq 0$, pour tout $x$, et $\psi_{(0)} \neq 0$ respectivement :

où

$$
(\lambda, \alpha) \stackrel{\omega}{\longrightarrow} \omega(\lambda, \alpha)=\left\{\omega_{x}(\lambda, \alpha)\right\}_{x \in A}
$$

$$
\begin{gathered}
\omega_{x}(\lambda, \alpha)=\lambda-\sum_{z \in \Lambda, z \neq x} H(x, z) \frac{\alpha(z)}{\alpha(x)} \\
(\lambda, \psi) \stackrel{h}{\longrightarrow}\{\lambda, \alpha(\psi), h(\psi)\}
\end{gathered}
$$

où $h(\psi)$ est une fonction régulière des $\psi(x), x \in \Lambda$, et $\alpha(x)=\frac{\psi(x)}{\psi(0)}$.

Ces deux applications vont nous permettre d'effectuer des changements de variable intéressants dans (VI.6). En fait, pour ces problèmes, il est naturel de travailler dans l'espace projectif et donc d'utiliser le changement de variable associé à $\omega$. Cependant, dans certaines situations il est préférable de conserver la symétrie entre tous les points de $\Lambda$, aussi on utilise le changement de variable $h \circ \omega$, en choisissant par exemple suivant les cas $h(\psi)=\|\psi\|^{2}$, ou bien $h(\psi)=\sum_{x \in A} \psi(x)$. Nous indiquerons donc les deux variantes. De tels changements de variable seraient peu efficaces si l'on ne pouvait en calculer le jacobien. C'est le but de notre

Théorème VI.1. Les jacobiens $J(\lambda, \alpha), J^{\prime}(\lambda, \psi)$ et $J^{\prime \prime}(\lambda, \psi)$ des applications $\omega$, h et $h \circ \omega$ peuvent s'exprimer par

$$
\begin{gathered}
J(\lambda, \alpha)=\|\alpha\|^{2} \sum_{T \in \mathscr{T}(\Lambda)}\left|\prod_{\ell \in T} H\left(x_{\ell}, y_{\ell}\right) \alpha\left(x_{\ell}\right) \alpha\left(y_{\ell}\right)\right| \prod_{x \in \Lambda}|\alpha(x)|^{-3} \\
J^{\prime}(\lambda, \psi)=|\psi(0)|^{-\Lambda}\left|\sum_{x \in \Lambda} \frac{\partial h}{\partial \psi(x)} \cdot \psi(x)\right| \\
J^{\prime \prime}(\lambda, \psi)=\|\psi\|^{2} \prod_{x \in \Lambda}|\psi(x)|^{-3}\left|\sum_{T \in \mathscr{T}(\Lambda)} \prod_{\ell \in T} H\left(x_{\ell}, y_{\ell}\right) \alpha\left(x_{\ell}\right) \alpha\left(y_{\ell}\right)\right| \\
\cdot\left|\sum_{x \in \Lambda} \frac{\partial h}{\partial \psi(x)} \cdot \psi(x)\right|
\end{gathered}
$$

où l'on somme sur les arbres maximaux ${ }^{14}$ de $\Lambda$, et où le premier produit porte sur toutes les lignes de l'arbre $T ; x_{\ell}$ et $y_{\ell}$ dénotent les extrémités de la ligne $\ell$.

14 C'est à dire les graphes connexes sans boucle construits sur tous les points de $A$ 
Preuve. Nous démontrerons seulement la formule (VI.13) pour le jacobien de $\omega$, la formule pour le jacobien $J^{\prime}(\lambda, \psi)$ se calculant facilement et la formule (VI.14) pour le jacobien $J^{\prime \prime}(\lambda, \psi)$ de $h \circ \omega$ se déduisant directement de (VI.13) et de l'expression de $J^{\prime}(\lambda, \psi)$. Le jacobien de $\omega$ est la valeur absolue du déterminant de la matrice $\left(a_{x y}\right)_{x \in \Lambda, y \in \Lambda}$, où au vu de (VI.10), (VI.11):

$$
a_{x y}=\frac{\partial \omega_{x}}{\partial \alpha(y)}=\delta_{x, y} \sum_{z \in A, z \neq x} H(x, z) \frac{\alpha(z)}{\alpha(x)^{2}}-H(x, y) \frac{1}{\alpha(x)}
$$

pour

$$
x \in \Lambda, y \in \Lambda \backslash 0
$$

et

$$
a_{x 0}=\frac{\partial \omega_{x}}{\partial \lambda}=1 \quad \text { pour } \quad x \in \Lambda
$$

Multiplions chaque ligne $x, x \in \Lambda$, par $\alpha(x)^{2}$ et chaque colonne $y, y \in \Lambda$, par $\alpha(y)$ (rappel $\alpha(0)=1$ ). Alors

$$
J(\lambda, \alpha)=\left|\operatorname{dét}\left(b_{x y}\right)_{x \in \Lambda, y \in \Lambda}\right| \prod_{x \in \Lambda}|\alpha(x)|^{-3}
$$

où

$$
b_{x y}=\delta_{x, y} \sum_{z \in \Lambda, z \neq x} H(x, z) \alpha(y) \alpha(z)-H(x, y) \alpha(x) \alpha(y) \quad \text { pour } \quad x \in \Lambda, y \in \Lambda \backslash 0
$$

et

$$
b_{x 0}=\alpha(x)^{2} \quad \text { pour } \quad x \in \Lambda
$$

Additionnant toutes les colonnes $x, x \in \Lambda$ à la colonne $x=0$, nous obtenons le déterminant d'une matrice $\left(b_{x y}^{\prime}\right)_{x \in \Lambda, y \in \Lambda}$ :

$$
\begin{array}{lll}
b_{x y}^{\prime}=b_{x y} & \text { pour } & x \in \Lambda, y \in \Lambda \backslash 0 \\
b_{x 0}^{\prime}=0 & \text { pour } & x \in \Lambda \backslash 0
\end{array}
$$

car selon (VI.18), $\sum_{x \in A} b_{x y}=0$ pour tout $y$; et enfin,

$$
b_{00}^{\prime}=\|\alpha\|^{2} \text {. }
$$

Nous pouvons alors développer ce déterminant selon la colonne $y=0$, ce qui conduit à

$$
J(\lambda, \alpha)=\|\alpha\|^{2}\left|\operatorname{dét}\left(b_{x y}\right)_{x \in \Lambda \backslash 0, y \in \Lambda \backslash 0}\right| \prod_{x \in \Lambda}|\alpha(x)|^{-3} .
$$

Mais le déterminant apparaissant dans l'équation (VI.23) est exactement le mineur d'un élément de la diagonale dans une matrice symétrique $\left(C_{x y}\right)_{x \in \Lambda, y \in \Lambda}$

$$
C_{x y}=\delta_{x, y} \sum_{z \in \Lambda, z \neq x} H(x, z) \alpha(x) \alpha(z)-H(x, y) \alpha(x) \alpha(y)
$$


pour tout $x \in \Lambda, y \in \Lambda$ matrice qui a la propriété que la somme de ses coefficients sur chaque ligne et chaque colonne est nulle. Le théorème de Kirchoff ([4] p. 48) nous exprime alors :

$$
\operatorname{dét}\left(b_{x y}\right)_{x \in \Lambda \backslash 0, y \in \Lambda \backslash 0}=\sum_{T \in \mathscr{T}(\Lambda)} \prod_{\ell \in T} b_{x_{\ell} y_{\ell}}
$$

Nous nous tournons maintenant vers le changement de variable annoncé permettant de calculer les observables à une énergie dans un volume $\Lambda$ fini. Nous énonçons:

Théorème VI.2. Supposons que le graphe formé par les couples $(x, y), x \in \Lambda, y \in \Lambda$ tels que $H(x, y) \neq 0$ soit un graphe connexe maximal ${ }^{15}$ de $\Lambda$. Alors pour toute fonction $g$, mesurable

et

$$
\int_{\mathbb{R} \Lambda} f_{g}^{\Lambda}(\omega) v_{H_{N D}}(\omega) d \omega=\int_{\mathbb{R} \Lambda} g(\lambda, \alpha) J(\lambda, \alpha) v_{H_{N D}}(\omega(\lambda, \alpha)) d \lambda d \alpha
$$

$\int_{\mathbb{R} A} f_{g}^{\Lambda}(\omega) v_{H_{N D}}(\omega) d \omega=\int_{\mathbb{R} \times \mathbb{R} \Lambda} g(\lambda, \psi) J^{\prime \prime}(\lambda, \psi) v_{H_{N D}}\left(\omega^{\prime}(\lambda, \psi)\right) \exp \{-h(\psi)\} d \lambda d \psi$

ò̀ $J$ et $J^{\prime \prime}$ sont exprimés dans la proposition ci-dessus.

Preuve. Nous démontrerons seulement (VI.26), la preuve de (VI.27) étant analogue dès que l'on a ajouté une intégration supplémentaire dans le membre de gauche $\int \beta(h) d h=1$, la fonction $\beta(h) \geqq 0$ étant arbitraire, et que nous avons choisi ici comme $\beta(h)=\exp \{-h\}$, afin que se présente naturellement le changement de variable de $\mathbb{R}^{|\Lambda|+1}$ dans $\mathbb{R}^{|\Lambda|+1}$ défini par $h \circ \omega$. Prouvons donc (VI.26).

Nous noterons $\Omega=\mathbb{R}^{|\Lambda|}$ l'espace des $\omega, A=\mathbb{R} \times \mathbb{R}^{|\Lambda|-1}$ l'espace des couples $(e, \alpha)$. Introduisons les ensembles suivants :

$$
S=\left\{\omega \in \mathbb{R}^{|\Lambda|} \mid H_{N D}^{(\Lambda)}+\omega \text { ait des valeurs propres dégénérées }\right\}
$$

$S$ est de mesure (de Lebesgue) nulle d'après la Proposition II.1.

$$
T=\left\{\omega \in \mathbb{R}^{|A|} \mid H_{N D}^{(A)}+\omega \text { ait un vecteur propre dont une composante soit nulle }\right\}
$$

$T$ est de mesure nulle d'après le Lemme VI.3 ci-après.

$$
T^{\prime}=\left\{\alpha \in \mathbb{R}^{|\Lambda|-1} \mid \exists x: \alpha(x)=0\right\}
$$

$\mathbb{R} \times T^{\prime}$ est de mesure nulle dans $A$.

La transformation $\omega$ est bien définie sur $A \backslash\left(\mathbb{R} \times T^{\prime}\right)$ qui est ouvert.

$$
S^{\prime}=\left\{(\lambda, \alpha) \in A \backslash \mathbb{R} \times T^{\prime} \mid \lambda \text { soit valeur propre dégénérée de } H_{N D}^{(\Lambda)}+\omega(\lambda, \alpha)\right\}
$$

$S^{\prime}$ sera montré plus loin de mesure nulle.

Nous compterons toujours dans ce qui suit les valeurs propres selon leur multiplicité. Définissons

$$
V_{i}=\left\{(\lambda, \alpha) \in A \backslash\left(\mathbb{R} \times T^{\prime} \cup S^{\prime}\right) \mid \lambda \text { soit la } i^{\text {ème }} \text { valeur propre de } H_{N D}^{(\Lambda)}+\omega(\lambda, \alpha)\right\} .
$$

15 C'est à dire un graphe connexe atteignant tous les points de $\Lambda$. Si telle n'est pas la situation, on considère les composantes connexes du graphe; l'opérateur $H^{(\Lambda)}$ se décompose en des $H_{j}^{(\Lambda)}$ associés à chacune de ces composantes, et l'on fait le changement de variable sur chacune d'entre elles 
$V_{i}$ est ouvert; en effet, soit $(\lambda, \alpha) \in V_{i}$ et soit $B$ une boule ouverte de centre $\omega(\lambda, \alpha): \omega$ étant continue sur $A \backslash \mathbb{R} \times T^{\prime}, \omega^{-1}(B)$ est un ouvert de $A \backslash \mathbb{R} \times T^{\prime}$, contenant $(\lambda, \alpha)$.

Choisissons $B$ assez petite de sorte que $\operatorname{Inf}_{\substack{j \neq i \\ \omega \in B}}\left|\lambda-\lambda_{j}(\omega)\right|>\eta$, ce qui est possible puisque $\lambda$ n'est pas dégénérée. Soit maintenant un ouvert $\mathcal{O}$ contenant $(\lambda, \alpha)$ tel que $\operatorname{Inf}_{\lambda^{\prime} \in A \backslash \mathcal{O}} d\left(\lambda, \lambda^{\prime}\right)>\eta / 2$. Alors l'ouvert $\omega^{-1}(B) \cap \mathcal{O}$ de $A \backslash \mathbb{R} \times T^{\prime}$ est inclus dans $V_{i}$.

Définissons alors

$$
V_{i} \ni(\lambda, \alpha) \stackrel{\omega_{2}}{\longrightarrow} \omega_{i}(\lambda, \alpha)=\omega(\lambda, \alpha) \in \Omega
$$

$\omega_{i}$ est un homéomorphisme analytique. Par ailleurs, les $V_{i}$ sont disjoints deux à deux et $\bigcup_{i=1}^{\Lambda} V_{i}=A \backslash\left(\mathbb{R} \times T^{\prime} \cup S^{\prime}\right)$. Cependant, nous avons

$$
\int_{\mathbb{R}^{|\Lambda|}} f_{g}^{\Lambda}(\omega) v_{H_{N D}}(\omega) d \omega=\sum_{i=1}^{|\Lambda|} \int_{\omega_{i}\left(V_{i}\right)} g\left(\lambda_{i}(\omega), \alpha_{i}(\omega)\right) v_{H_{N D}}(\omega) d \omega
$$

où $\lambda_{i}(\omega), \alpha_{i}(\omega)$ désignent la $i^{\text {ème }}$ valeur propre et son vecteur propre associé pour $\omega \in \omega_{i}\left(V_{i}\right)$; (VI.28) est vraie parce que $\forall i, \Omega \backslash \omega_{i}\left(V_{i}\right) \subset T \cup S$ qui est de mesure nulle (Lemme II.1 et Lemme V.3 ci-après).

Maintenant dans (VI.28) nous pouvons effectuer le changement de variable associé à $\omega_{i}$ et:

$$
\int_{\mathbb{R}} \int_{\Delta \mid} f_{g}^{\Lambda}(\omega) v_{I I_{N} D}(\omega) d \omega=\int_{\substack{\mid \bigcup_{i=1}^{A} V_{i} \\ V_{i}}} g(\lambda, \alpha) J(\lambda, \alpha) v_{H_{N D}}(\omega(\lambda, \alpha)) d \lambda d \alpha
$$

Sachant que $\bigcup_{i=1}^{A} V_{i}=A \backslash\left(\mathbb{R} \times T^{\prime} \cup S^{\prime}\right)$, et comme $\mathbb{R} \times T^{\prime}$ est de mesure nulle, il ne reste plus qu'à montrer que $S^{\prime}$ est de mesure nulle. Pour cela si $\chi(A)$ désigne la fonction caractéristique de l'ensemble $A$, considérons

$$
\begin{gathered}
\int_{A \backslash \mathbb{R} \times T^{\prime}} \chi\left(S^{\prime}\right) J(\lambda, \alpha) v_{H_{N D}}(\omega(\lambda, \alpha)) d \lambda d \alpha . \leqq \int_{A \backslash \mathbb{R} \times T^{\prime}} \chi(\omega(\lambda, \alpha) \in S) \\
\cdot J(\lambda, \alpha) v_{H_{N D}}(\omega(\lambda, \alpha)) d \lambda d \alpha \leqq|\Lambda| \int_{\Omega} \chi(S) v_{H_{N D}}(\omega) d \omega=0
\end{gathered}
$$

la dernière inégalité étant obtenue de la façon suivante: on considère l'application (1) de $A \backslash \mathbb{R} \times T^{\prime}$ dans $\Omega$ qui définit une transformation de classe $C^{1}$ (et même analytique) mais non inversible. Soit $c(\omega)$ le nombre de points de l'image inverse d'un point de $\Omega$ par cette application; $c(\omega)$ est donc le nombre de couples $(\lambda, \alpha)$ tels que $\alpha(x) \neq 0, \forall x \in \Lambda$, et donc $c(\omega) \in\{0,1, \ldots,|\Lambda|\} \cup\{\infty\}$. De plus, $c(\omega)=|\Lambda|$ presque partout (Lemme II.1). En appliquant le théorème de changement de variable pour de telles applications [27] on obtient (VI.30).

Ceci clôt la démonstration du Théorème VI.2. Il ne reste plus qu'à prouver le fait que nous avons admis ci-dessus, que $T$ est un ensemble de mesure nulle, ce qui est le but du Lemme VI.3.

Lemme VI.3. Supposons que le graphe formé par les couples $(x, y), x \in \Lambda, y \in \Lambda$ tels que $H(x, y) \neq 0$, soit un graphe connexe maximal de $\Lambda$. Alors l'ensemble $T=\left\{\omega \in \mathbb{R}^{|\Lambda|} \mid \exists \lambda \in \mathbb{R}, \alpha \in \mathbb{P}^{|\Lambda|-1}:\left(H_{N D}^{\Lambda}+\omega\right) \alpha=\lambda \alpha\right.$ et $\left.\exists z \in \Lambda, \alpha(z)=0\right\}$ est de mesure nulle. 
Preuve. La matrice diagonale $\omega$ sera ici appelée $\omega^{4}$ afin de spécifier les variables dont elle dépend. Remarquons qu'en prenant pour nouvelles variables $\omega_{x}$ les variables $\omega_{x}-\lambda$, il suffit de montrer que

$$
T_{\Lambda}=\left\{\omega \in \mathbb{R}^{|\Lambda|} \mid \exists \alpha \in \mathbb{P}^{|A|-1},\left(H_{N D}^{\Lambda}+\omega^{\Lambda}\right) \alpha=0, \quad \text { et } \quad \exists z \in \Lambda, \alpha(z)=0\right\}
$$

est inclus dans un ensemble analytique de codimension 2. Mais

où

$$
T_{\Lambda}=\bigcup_{z \in \Lambda} T_{\Lambda, z}
$$

$$
\begin{aligned}
T_{\Lambda, z} & =\left\{\omega \in \mathbb{R}^{|\Lambda|} \mid \exists \alpha \in \mathbb{P}^{|\Lambda|-1},\left(H_{N D}^{\Lambda}+\omega^{\Lambda}\right) \alpha=0 \quad \text { et } \quad \sum_{y \in \Lambda \mid\{z\}} H(z, y) \alpha(y)=0\right\} \\
& =\left\{\omega \in \mathbb{R}^{|\Lambda|} \mid \exists \alpha^{\prime} \in \mathbb{P}^{|\Lambda|-2},\left(H_{N D}^{A \mid\{z\}}+\omega^{\Lambda \backslash\{z\}}\right) \alpha^{\prime}=0 \quad \text { et } \sum_{y \in \Lambda \backslash\{z\}} H(z, y) \alpha^{\prime}(y)=0\right\} .
\end{aligned}
$$

Considérons un point $x$ de $\Lambda \backslash\{z\}$ tel que $H(z, x) \neq 0$. Il en existe, en raison de l'hypothèse du lemme. Nous voyons alors que

$$
T_{A, z}=\left\{\omega \in \mathbb{R}^{|A|} \mid \Delta_{A \backslash\{z\}}(\omega)=0 \quad \text { et } \quad Q_{A \backslash\{z\}}^{x}(\omega)=0\right\}
$$

où $\Delta_{A}(\omega)$ désigne le déterminant de $H_{N D}^{(A)}+\omega^{(A)}$, et $Q_{A}^{x}(\omega)$ le déterminant où l'on a remplacé dans le précédent la ligne $x$ par la ligne $\{H(z, y)\}_{y \in A}$ associée à l'équation

$$
\sum_{y \in A} H(z, y) \alpha(y)=0 .
$$

Comme

$$
\Delta_{\Lambda \backslash\{z\}}(\omega)=\omega_{x} \Delta_{\Lambda \mid\{z, x\}}(\omega)+R_{\Lambda \backslash\{z, x\}}(\omega)
$$

où $R$ ne dépend pas de $\omega_{x}$, nous voyons que

$$
\begin{aligned}
& T_{A, z}=T_{\Lambda, z}^{\prime} \cup T_{\Lambda, z}^{0} \\
& T_{A, z}^{\prime}=\left\{\omega \in \mathbb{R}^{|\Lambda|} \mid \Delta_{\Lambda \backslash\{z, x\}}(\omega) \neq 0, Q_{\Lambda \backslash\{z\}}^{x}(\omega)=0 \quad \text { et } \quad \omega_{x}=-\frac{R_{A \backslash\{z, x\}}(\omega)}{\Delta_{\Lambda \backslash\{z, x\}}(\omega)}\right\} \\
& T_{\Lambda, z}^{0}=\left\{\omega \in \mathbb{R}^{|A|} \mid \Delta_{\Lambda \backslash\{z, x\}}(\omega)=0, \Delta_{\Lambda \backslash\{z\}}(\omega)=0 \quad \text { et } \quad Q_{\Lambda \backslash\{z\}}^{x}(\omega)=0\right\}
\end{aligned}
$$

$Q_{\Lambda \backslash\{z\}}^{x}(\omega)$ ne dépend pas de $\omega_{x}$ et son terme d'ordre maximal dans les $\omega$ est $H(x, z) \prod_{y \in \Lambda \backslash\{z, x\}} \omega_{y}$, et comme $H(x, z)$ est non nul, $Q$ n'est pas identiquement nul. Il en suit que $T_{A, z}^{\prime}$ est un ensemble analytique de codimension 2. Par ailleurs, le cofacteur du terme de position $(x, x)$ dans $Q_{\Lambda \backslash\{z\}}^{x}(\omega)$ est $\Delta_{\Lambda \backslash\{z, x\}}(\omega)$, qui est nul dans $T_{A, z^{\prime}}^{0}$ On peut donc remplacer ce terme qui est $H(x, z)$ par 0 dans $Q$, obtenant ainsi $Q_{\Lambda \backslash\{z\}}^{\prime \prime 2}(\omega)$, et

$$
T_{\Lambda, z}^{0} \subset\left\{\omega \in \mathbb{R}^{|\Lambda|} \mid \Delta_{\Lambda \mid\{z\}}(\omega)=0 \quad \text { et } \quad Q_{\Lambda \backslash\{z\}}^{\prime x}(\omega)=0\right\} .
$$

Mais on voit alors que ce dernier ensemble n'est autre que l'ensemble $T_{A, z}$, mais associé à une matrice $H_{N D}^{(A)}$ dans laquelle on aurait posé $H(x, z)=H(z, x)=0$.

On procède alors par récurrence, supprimant des lignes entre $z$ et $\Lambda \backslash\{z\}$. Lorsque l'on supprime la dernière de ces lignes, on est ramené à un problème semblable au problème initial mais dans une boîte $\Lambda \backslash\{z\}$ au lieu de $\Lambda$. Ainsi on diminue le nombre de points de $|\Lambda|$, jusqu'à aboutir à un problème trivial. 


\section{B. L'opérateur de Schrödinger unidimensionnel}

Dans les sections qui suivent nous étudierons le cas particulier de l'opérateur de Schrödinger unidimensionnel discrétisé avec un potentiel aléatoire distribué selon une loi produit. C'est-à-dire que l'ensemble $S$ sera identifié à $\mathbb{Z}$ ou $\mathbb{N}$ et que la mesure $\mu$ est choisie de sorte que avec probabilité 1

$$
\begin{array}{llll}
H(x, y)=0 & \text { si } & |x-y| \neq 1 \\
H(x, y)=-1 & \text { si } & |x-y|=1, \quad x \in S, y \in S
\end{array}
$$

et les termes diagonaux

$$
\left\{\omega_{x}\right\}_{x \in S}=\{H(x, x)\}_{x \in S}
$$

sont indépendants et distribués selon des lois absolument continues identiques ${ }^{16}$ de densité commune $r(\omega)$.

En d'autres termes nous considérons l'opérateur aléatoire $H(\omega)$ défini par

$$
(H \psi)(x)=-\psi(x+1)-\psi(x-1)+\omega_{x} \psi(x)
$$

où les $\omega_{x}$ sont des variables aléatoires indépendantes de densité $r(\omega)$. On notera que dans les définitions physiques, le potentiel $V_{x}$ est donné par $V_{x}=\omega_{x}-2$.

\section{Fonction spectrale moyenne et fonction de corrélation à volume fini}

Nous entamons ici l'étude des fonctions de corrélation. Nous voulons obtenir des expressions utiles, pour leur analyse ultérieure, de la fonction spectrale moyenne et de la fonction de corrélation. Puisque $S$ est la droite ou la demi-droite, nous pourrons sans restriction choisir des boîtes $\Lambda$ de la forme $\Lambda=[-M, \ldots,+N]$, la limite thermodynamique consistant à laisser $M$ ou $N$, ou bien $M$ et $N$ tendre vers l'infini.

Si $H^{A}(\omega)$ est la restriction de l'opérateur $H(\omega)$ à la boîte $\Lambda$, rappelons que (en utilisant la Proposition II.1)

et

$$
\varrho_{\mu}^{\Lambda}\left(x, x^{\prime} ; A\right)=\int_{\mathbb{R}^{\prime}|\Lambda|} \prod_{y \in \Lambda} r\left(\omega_{y}\right) d \omega_{y}\left\{\sum_{\lambda \in A} \psi_{\lambda}(x) \psi_{\lambda}\left(x^{\prime}\right)\right\}
$$

$$
\bar{\varrho}_{\mu}^{\Lambda}\left(x, x^{\prime} ; A\right)=\int_{\mathbb{R}^{\prime}|\Lambda|} \prod_{y \in \Lambda} r\left(\omega_{y}\right) d \omega_{y}\left\{\sum_{\lambda \in A}\left|\psi_{\lambda}(x)\right|\left|\psi_{\lambda}\left(x^{\prime}\right)\right|\right\}
$$

où pour $\omega$ fixé tel que $H^{A}(\omega)$ n'ait pas de valeur propre dégénérée, la somme porte sur les valeurs propres de $H^{A}(\omega)$ dans l'intervalle $A$ et $\psi_{\lambda}$ désigne alors le vecteur propre normalisé, associé à $\lambda$, de $H^{A}(\omega)$.

Il apparaît intéressant d'étudier des fonctions plus générales, où le potentiel $\omega_{x}$ au bord de la boîte n'est plus distribué selon $r(\omega)$, mais selon une distribution $\beta(\omega)$ en $-M$ et $\delta(\omega)$ en $N$. Ceci revient en fait à prendre des densités $r(\omega)$ dépendant de $x$ et $\Lambda$. Nous introduisons cette variante afin d'étudier si les fonctions $\varrho^{\Lambda}$ et $\bar{\varrho}^{\Lambda}$ dépendent dans la limite thermodynamique d'une telle perturbation aux bords.

16 En fait une large partie de ces sections ne nécessite pas que les lois soient identiques. En particulier il est d'intérêt de pouvoir choisir des distributions $r^{\prime}(\omega)$ différentes sur les bords de la boîte afin d'étudier la dépendance par rapport à ces conditions aux bords 
Pour alléger les notations, nous n'écrirons pas la dépendance en $\beta$ et $\delta$ explicitement sauf lorsqu'elle sera nécessaire.

C'est dans l'application maintenant de notre formalisme général de la Sect. VI qu'apparaît la simplification essentielle associée au cas de l'opérateur de Schrödinger unidimensionnel avec des conditions aux bords vides: dans le jacobien du changement de variable, un seul arbre maximal apparaît.

Proposition VII.1. Soit $\Lambda=\{-M, \ldots,+N\}$ et $x \leqq x^{\prime} \in \Lambda$. Alors :

$$
\begin{aligned}
\bar{\varrho}_{\mu}^{\Lambda}\left(x, x^{\prime} ; A\right)= & \frac{1}{2} \int_{A} d \lambda \int_{\mathbb{R}^{N+M}}\left|\alpha(x) \alpha\left(x^{\prime}\right)\right| \cdot\left|\alpha(-M)^{-1} \alpha(N)^{-1}\right| \\
& \cdot \prod_{z \in A}\left\{|\alpha(z)|^{-1} r_{\lambda}^{(z)}\left(\frac{\alpha(z+1)+\alpha(z-1)}{\alpha(z)}\right)\right\} d \alpha
\end{aligned}
$$

ò̀ $\quad \alpha(-M-1)=\alpha(N+1)=0, \quad \alpha(-M)=1, \quad d \alpha=\prod_{-M<x \leqq N} d \alpha(x), \quad r_{\lambda}(u)=r(\lambda-u)$. $r^{(-M)}=\beta, r^{(N)}=\delta$ et $r^{(z)}=r$ pour $-M<z<N$.

En posant:

$$
\bar{\varrho}_{\mu}^{\Lambda}\left(x, x^{\prime} ; A\right)=\int_{A} \bar{\varrho}_{\mu}^{\Lambda}\left(x, x^{\prime} ; \lambda\right) d \lambda
$$

nous avons aussi

$$
\begin{gathered}
\bar{\varrho}_{\mu}^{\Lambda}\left(x, x^{\prime} ; \lambda\right)=\frac{1}{2} \int_{\mathbb{R}^{N+M}} \prod_{x \leqq z \leqq x^{\prime}-1}\left|y_{z}\right|^{-1} \prod_{x^{\prime} \leqq z \leqq N-1}\left|y_{z}\right|^{-2} \\
\prod_{-M+1 \leqq z \leqq+N-1} r_{\lambda}\left(y_{z-1}^{-1}+y_{z}\right) \beta_{\lambda}\left(y_{-M}\right) \delta_{\lambda}\left(y_{N-1}^{-1}\right) \prod_{-M \leqq z \leqq N-1} d y_{z}
\end{gathered}
$$

où par convention $\prod_{z \in \emptyset} f(z)=1$.

La fonction spectrale moyenne $\varrho_{\mu}^{\Lambda}\left(x, x^{\prime} ; A\right)$ et la quantité associée $\varrho_{\mu}^{\Lambda}\left(x, x^{\prime} ; \lambda\right)$ sont données par des formules analogues à (VII.3), (VII.5), dans lesquelles on remplace $\left|\alpha(x) \alpha\left(x^{\prime}\right)\right|$ par $\alpha(x) \alpha\left(x^{\prime}\right)$ et

$$
\prod_{x \leqq z \leqq x^{\prime}-1}\left|y_{z}\right|^{-1} \text { par } \prod_{x \leqq z \leqq x^{\prime}-1} y_{z}^{-1} .
$$

Preuve. La première partie de la proposition [formule (VII.3)] se déduit directement des Théorèmes VI.1 et VI.2, en utilisant les hypothèses spécifiques à cette partie, et en choisissant de faire la projection stéréographique au point $-M$ au lieu du point 0 . En particulier nous avons utilisé le fait que pour le cas unidimensionnel, en utilisant les conditions aux bords vides ${ }^{17}$ pour $H_{A}$, le graphe des $\{H(x, y)\}$ ne contient qu'un seul arbre maximal. C'est là, au moins dans notre formalisme une des simplifications essentielles qu'apporte la dimension 1.

La seconde partie, la formule (VII.5) se déduit de la précédente en introduisant les variables

$$
y_{z}=\frac{\alpha(z+1)}{\alpha(z)}, \quad-M \leqq z \leqq N-1
$$

le jacobien du changement de variables $\alpha \rightarrow y$ étant $\prod_{-M \leqq z<N}|\alpha(z)|$.

17 Contrairement à toutes les sections précédentes où $H_{A}$ pouvait être défini avec d'autres conditions aux bords, périodiques, antipériodiques ..., sans entraîner de difficultés supplémentaires, il est ici plus simple de travailler avec les conditions aux bords vides 
Nous introduisons maintenant un opérateur qui va jouer un rôle fondamental dans la suite de ce papier:

Définition. Nous appellerons opérateur de transfert ${ }^{18}$ l'opérateur intégral $T_{\varepsilon, \lambda}$ de noyau

$$
T_{\varepsilon, \lambda}\left(y^{\prime} \mid y\right)=\left|y^{\prime}\right|^{-\varepsilon} r_{\lambda}\left(y^{\prime}+y^{-1}\right) .
$$

Nous introduisons aussi l'opérateur intégral $\stackrel{\circ}{T}_{1, \lambda}$ de noyau

$$
\stackrel{\circ}{T}_{1, \lambda}\left(y^{\prime} \mid y\right)=y^{\prime-1} r_{\lambda}\left(y^{\prime}+y^{-1}\right) .
$$

Utilisant l'opérateur $T$, nos corrélation et fonction spectrale moyenne peuvent se réécrire sous la forme suivante, qui conduira dans les sections ultérieures à des formules explicites pour leurs limites:

\section{Proposition VII.2.}

$$
\begin{aligned}
& \varrho_{\mu}^{\Lambda}\left(x, x^{\prime} ; \lambda\right)=\frac{1}{2} \int_{\mathbb{R}}\left(\stackrel{T}{T}_{1, \lambda}^{x^{\prime}-x} T_{0, \lambda}^{M+x} \beta_{\lambda}\right)(y)\left(T_{0, \lambda}^{N-1-x^{\prime}} \delta_{\lambda}\right)\left(y^{-1}\right) d y, \\
& \bar{\varrho}_{\mu}^{\Lambda}\left(x, x^{\prime} ; \lambda\right)=\frac{1}{2} \int_{\mathbb{R}}\left(T_{1, \lambda}^{x^{\prime}-x} T_{0, \lambda}^{M+x} \beta_{\lambda}\right)(y)\left(T_{0, \lambda}^{N-1-x^{\prime}} \delta_{\lambda}\right)\left(y^{-1}\right) d y .
\end{aligned}
$$

L'utilité de l'opérateur $T_{\varepsilon, \lambda}$ introduit plus haut vient de l'inégalité suivante

Proposition VII.3. Pour tout $\varepsilon, 0 \leqq \varepsilon \leqq 1$, nous avons

$$
\begin{aligned}
\left|\varrho_{\mu}^{\Lambda}\left(x, x^{\prime} ; \lambda\right)\right| \leqq & \varrho_{\mu}^{\Lambda}\left(x, x^{\prime} ; \lambda\right) \leqq \frac{1}{2} \int_{\mathbb{R}}\left(T_{\varepsilon, \lambda}^{x^{\prime}-x} T_{0, \lambda}^{M+x} \beta_{\lambda}\right)(y) \\
& \cdot\left(T_{0, \lambda}^{N-1-x^{\prime}} \delta_{\lambda}\right)\left(y^{-1}\right) d y+\frac{1}{2} \int_{\mathbb{R}}\left(T_{\varepsilon, \lambda}^{x^{\prime}-x} T_{0, \lambda}^{N-x^{\prime}} \delta_{\lambda}\right)\left(y^{-1}\right) \\
& \cdot\left(T_{0, \lambda}^{M+x-1} \beta_{\lambda}\right)(y) d y .
\end{aligned}
$$

Preuve. L'inégalité (VII.10) se dérive rapidement de la formule (VII.8) en $y$ introduisant la décomposition

$$
\begin{aligned}
\prod_{1 \leqq z \leqq x^{\prime}-x}\left|y_{z}\right|^{-1}= & \prod_{1 \leqq z \leqq x^{\prime}-x}\left|y_{z}\right|^{-1} \theta\left(\left|y_{1} \ldots y_{x^{\prime}-x}\right| \geqq 1\right) \\
& +\prod_{1 \leqq z \leqq x^{\prime}-x} \frac{\left|y_{z}\right|}{\left|y_{z}\right|^{2}} \theta\left(\left|y_{1} \ldots y_{x^{\prime}-x}\right| \leqq 1\right)
\end{aligned}
$$

où $\theta$ est la fonction de Heavyside.

L'égalité (VII.11) permet en fait de symmétriser (VII.8) par rapport à la gauche et la droite, si l'on fait le changement de variable $y \rightarrow y^{-1}$ dans le second terme.

L'inégalité (VII.10) souhaitée se déduit par (VII.11) en remarquant que

$$
\begin{aligned}
\prod_{1 \leqq z \leqq x^{\prime}-x}\left|y_{z}\right|^{-1} \leqq & \prod_{1 \leqq z \leqq x^{\prime}-x}\left|y_{z}\right|^{-\varepsilon} \theta\left(\left|y_{1} \ldots y_{x^{\prime}-x}\right| \geqq 1\right) \\
& +\prod_{1 \leqq z \leqq x^{\prime}-x} \frac{\left|y_{z}\right|^{+\varepsilon}}{\left|y_{z}\right|^{2}} \theta\left(\left|y_{1} \ldots y_{x^{\prime}-x}\right| \leqq 1\right)
\end{aligned}
$$

et en faisant le changement de variable $y \rightarrow y^{-1}$ dans le second terme.

Il est également intéressant de calculer le rapport de participation inverse, et plus généralement ce que nous avons appelé la mesure de transfert de $\mu$ entre deux

18 Par référence à la matrice de transfert en mécanique statistique 
points $x$ et $y$, soit

$$
R_{\mu}^{\Lambda}(x, y ; A)=\mu\left(\sum_{\lambda \in \operatorname{Sp} H^{\Lambda} \cap A} \psi_{\lambda}^{2}(x) \psi_{\lambda}^{2}(y)\right)
$$

Nous étudierons à nouveau le cas où le potentiel est distribué selon $r(\cdot)$ à l'intérieur de $\Lambda=[-M, N]$, mais selon $\beta(\cdot)$ au point $-M$ et selon $\delta$ au point $N$.

Pour cela nous allons de nouveau utiliser notre formalisme général décrit dans la Sect. VI, mais cette fois nous utiliserons le changement de variable (VI.29) dans le Théorème VI.2:

$$
(\omega, h) \rightarrow(\psi, \lambda)
$$

$\operatorname{avec} h(\psi)=\sum_{x \in \Lambda} \psi^{2}(x)=\|\psi\|^{2}$.

Nous obtenons alors en vertu de (VI.29)

$$
\begin{aligned}
R_{\mu}^{A}(x, y ; A)= & \int_{A} d \lambda \int \frac{d \psi(-M)}{\psi^{2}(-M)} \beta_{\lambda}\left(\frac{\psi(-M+1)}{\psi(-M)}\right) \\
& \cdot \prod_{z=-M+1}^{N-1}\left[r_{\lambda}\left(\frac{\psi(z-1)+\psi(z+1)}{\psi(z)}\right) \frac{d \psi(z)}{|\psi(z)|}\right] \\
& \cdot \delta_{\lambda}\left(\frac{\psi(N-1)}{\psi(N)}\right) \frac{d \psi(N)}{\psi^{2}(N)} \exp \left\{-\sum_{z=-M}^{N} \psi^{2}(z)\right\} \psi^{2}(x) \psi^{2}(y)
\end{aligned}
$$

Cette expression peut se mettre sous la forme plus commode suivante.

Proposition VII.4. Soit $\Lambda=[-M, N]$ et $x, y \in \Lambda,-M<x \leqq y<N$. Alors

$$
\begin{aligned}
R^{\Lambda}(x, y ; A)= & \int_{A} d \lambda \int_{\mathbb{R}^{y-x+3}} \frac{\psi^{2}(0) \psi^{2}(y-x)}{|\psi(-1) \psi(y-x+1)|} \\
& \cdot a_{N-y-1}\left(\psi(y-x), \psi(y-x+1) \mid \delta_{\lambda}\right) \\
& \cdot a_{M+x-1}\left(\psi(0), \psi(-1) \mid \beta_{\lambda}\right) \\
& \cdot \prod_{0 \leqq z \leqq y-x} r_{\lambda}\left(\frac{\psi(z-1)+\psi(z+1)}{\psi(z)}\right) \\
& \cdot \prod_{-1 \leqq z \leqq y-x+1}\left\{e^{-\psi^{2}(z)} \frac{\psi(z)}{|\psi(z)|}\right\}
\end{aligned}
$$

$o \grave{u}$

$$
\begin{aligned}
a_{n}(\psi(0), \psi(+1) \mid f)= & \int_{\mathbb{R}^{n}} \frac{|\psi(1)|}{|\psi(n+1)|} f\left(\frac{\psi(n)}{\psi(n+1)}\right) \prod_{1 \leqq z \leqq n} r_{\lambda}\left(\frac{\psi(z-1)+\psi(z+1)}{\psi(z)}\right) \\
& \cdot \prod_{1<z \leqq n+1}\left\{e^{-\psi(z)^{2}} \frac{d \psi(z)}{|\psi(z)|}\right\} \\
= & \int_{\mathbb{R}^{n}} f\left(y_{1}\right) \prod_{1 \leqq z \leqq n}\left\{r_{\lambda}\left(y_{z+1}+y_{z}^{-1}\right)\right. \\
& \left.\cdot e^{-\psi(1)^{2} \mid y_{n} \ldots y_{n-z+\left.1\right|^{-2}}} d y_{z}\right\} \\
\text { et } y_{n+1}=\frac{\psi(0)}{\psi(1)} &
\end{aligned}
$$


Preuve. Ce résultat s'obtient en séparant dans (VII.13) les intégrations en $\psi(z)$ pour $x-1 \leqq z \leqq y+1$ et les autres et en relabellant les variables de façon naturelle; la réécriture (VII.16) de (VII.15) est obtenue en $y$ faisant le changement de variable $y_{k}=\frac{\psi(n-k+1)}{\psi(n-k+2)}, k=1, \ldots, n$.

\section{Etude de l'opérateur de transfert}

Il apparaît clairement dans la section précédente que les propriétés de l'opérateur $T_{\varepsilon}$, ou plutôt de la famille $T_{\varepsilon}$, vont être déterminantes. Nous baptiserons par simplicité l'opérateur $T_{\varepsilon}$ «opérateur de transfert».

Nous ferons désormais une hypothèse sur la densité $r(x)$. Nous supposerons qu'elle appartient à $L_{\infty}\left((1+|x|)^{1+\alpha}\right)$ pour un certain $\alpha, 0<\alpha<1$, c'est-à-dire à l'espace des fonctions $f$ :

$$
L_{\infty}\left((1+|x|)^{1+\alpha}\right)=\left\{f\left|\operatorname{Sup}_{x \in \mathbb{R}}\right| f(x)(1+|x|)^{1+\alpha} \mid<\infty\right\} .
$$

En particulier $r(\cdot)$ appartient donc à $L_{\infty}$. Cette hypothèse n'est guère contraignante physiquement: les physiciens choisissent pour $r(x)$ généralement la fonction caractéristique d'un intervalle, ou une gaussienne, ou une lorentzienne, toutes densités qui sont dans la classe considérée.

Par ailleurs, $r(x)$ est une densité et satisfait donc:

Nous introduisons aussi la fonction $\sigma$ :

$$
r(x) \geqq 0 \quad \text { et } \quad \int_{\mathbb{R}} r(x) d x=1 .
$$

$$
\sigma(x)=|x|^{+1-\alpha / 2}(1+|x|)^{+\alpha} .
$$

Les notations $B(\mathbb{R}, \sigma)$ et $C(\overline{\mathbb{R}}, \sigma)$ désigneront respectivement l'espace des fonctions à valeurs complexes qui avec le poids $\sigma$ sont bornées ou continues sur $\overline{\mathbb{R}}$ le compactifié de $\mathbb{R}$, c'est-à-dire

$$
\begin{gathered}
B(\mathbb{R}, \sigma)=\left\{f\left|\operatorname{Sup}_{x \in \mathbb{R}}\right| f(x) \mid \sigma(x)<\infty\right\} \\
C(\overline{\mathbb{R}}, \sigma)=\{f \mid f(x) \sigma(x) \text { soit continue sur } \overline{\mathbb{R}}\} .
\end{gathered}
$$

Nous introduisons aussi l'espace $\mathscr{M}\left(\overline{\mathbb{R}}, \sigma^{-1}\right)$, espace des mesures de Baire sur l'espace compact $\overline{\mathbb{R}}$ avec le poids $\sigma^{-1}$, qui est le dual de $C(\overline{\mathbb{R}}, \sigma)$ :

Nous pouvons alors énoncer:

$$
\mathscr{M}\left(\overline{\mathbb{R}}, \sigma^{-1}\right)=C(\overline{\mathbb{R}}, \sigma)^{*} .
$$

Proposition VIII.1. Supposons que la densité $r$ soit dans $L_{\infty}\left((1+|x|)^{1+\alpha}\right)$ et soit $\varepsilon \in \mathbb{C}$, $|\operatorname{Re} \varepsilon|<\inf (1-\alpha / 2, \alpha / 2)=\varepsilon_{0}$, et $\lambda \in \mathbb{R}$. Alors :

a) $T_{\varepsilon, \lambda}$ envoie $B(\mathbb{R}, \sigma)$ et donc aussi $C(\overline{\mathbb{R}}, \sigma)$ dans $C(\overline{\mathbb{R}}, \sigma)$.

b) $\forall f \in B(\mathbb{R}, \sigma),\left|\left(T_{\varepsilon, \lambda} f\right)(x)\right|=0(1+|x|)^{-s}, s=1+\alpha+\operatorname{Re} \varepsilon$.

c) $T_{\varepsilon, \lambda}$ est un opérateur compact de $C(\mathbb{R}, \sigma)$ dans lui-même.

d) $T_{\varepsilon, \lambda}$ est une famille analytique en $\varepsilon$.

e) $T_{\varepsilon, \lambda}$ est continu en $\lambda$, $\varepsilon$.

f) L'adjoint $T_{\varepsilon, \lambda}^{*}$ de $T_{\varepsilon, \lambda}$ satisfait des propriétés analogues, et en particulier est un opérateur compact de $\mathscr{M}\left(\overline{\mathbb{R}}, \sigma^{-1}\right)$ dans lui-même. 
Preuve. Il est naturel d'introduire l'opérateur intégral $\mathscr{K}_{\varepsilon, \lambda}$ de noyau

$$
\begin{aligned}
\mathscr{K}_{\varepsilon, \lambda}(x \mid y) & =\sigma(x) T_{\varepsilon, \lambda}(x \mid y) \sigma^{-1}(y) \\
& =\sigma(x)|x|^{-\varepsilon} r_{\lambda}\left(x+y^{-1}\right) \sigma^{-1}(y) .
\end{aligned}
$$

Nous montrons d'abord un résultat technique, des propriétés de base du noyau $\mathscr{K}_{\varepsilon}(x \mid y)$ dans le

Lemme VIII.2. Pour tout $\lambda \in \mathbb{R}$ et $\varepsilon,|\operatorname{Re} \varepsilon|<\varepsilon_{0}, \mathscr{K}_{\varepsilon, \lambda}(x \mid y)$ satisfait

$$
\int_{\mathbb{R}}\left|\mathscr{K}_{\varepsilon, \lambda}(x \mid y)\right| d y<\frac{C(\varepsilon, \lambda, r)}{(1+|x|)^{\alpha / 2+\operatorname{Re} \varepsilon}}
$$

où $C(\varepsilon, \lambda, r)$ est une constante, et

$$
\forall x \in \mathbb{R}, \lim _{h \rightarrow 0} \int\left|\mathscr{K}_{\varepsilon, \lambda}(x+h \mid y)-\mathscr{K}_{\varepsilon, \lambda}(x \mid y)\right| d y=0 .
$$

Preuve. En utilisant les définitions et hypothèses, on voit que

$$
\begin{aligned}
& \int_{\mathbb{R}}\left|\mathscr{K}_{\varepsilon, \lambda}(x \mid y)\right| d y \leqq|x|^{1-\alpha / 2}(1+|x|)^{\alpha}|x|^{-\operatorname{Re} \varepsilon} \\
& \cdot \int \frac{A(r, \lambda)}{\left(1+\left|x+y^{-1}\right|\right)^{1+\alpha}}|y|^{-1+\alpha / 2}(1+|y|)^{-\alpha} d y
\end{aligned}
$$

où $A$ est une constante ne dépendant que de $r$ et de $\lambda$. On en déduit (VIII.4). Quant à (VIII.5) il s'obtient par le théorème de Lebesgue lorsque $r$ et donc $\mathscr{K}_{\varepsilon, \lambda}$ est une fonction continue. Si ce n'est pas le cas, on peut approximer $r$ par une fonction continue et appliquer alors Lebesgue.

Nous revenons donc à la preuve de la Proposition VIII.1. Les résultats de ce lemme ci-dessus assurent que $\mathscr{K}_{\varepsilon, \lambda}$ envoie $B(\mathbb{R})$ et $C(\overline{\mathbb{R}})$ dans $C(\overline{\mathbb{R}})$ et qu'il est compact d'où les résultats $\mathrm{a}), \mathrm{b}$ ) et $\mathrm{c}$ ) de la Proposition VIII.1.

Montrons maintenant que $T_{\varepsilon, \lambda}$ forme une famille analytique en $\varepsilon$. Introduisons $T_{\varepsilon, \lambda}^{\prime}$ de noyau

$$
T_{\varepsilon, \lambda}^{\prime}(x \mid y)=-T_{0, \lambda}(x \mid y)|x|^{-\varepsilon} \ln |x| .
$$

Par des estimations directes, on voit que lorsque $|\varepsilon|<\varepsilon_{0}$

$$
\begin{gathered}
\lim _{\varepsilon^{\prime} \rightarrow \varepsilon} \operatorname{Sup}_{x \in \mathbb{R}} \sigma(x) \int\left|\frac{T_{\varepsilon, \lambda}(x \mid y)-T_{\varepsilon^{\prime}, \lambda}(x \mid y)}{\varepsilon-\varepsilon^{\prime}}-T_{\varepsilon^{\prime}}^{\prime}(x \mid y)\right| \sigma^{-1}(y) d y \\
\leqq \lim _{\varepsilon^{\prime} \rightarrow \varepsilon} \operatorname{Sup}_{x \in \mathbb{R}} \sigma(x)|x|^{-\operatorname{Re} \varepsilon\left|\varepsilon^{\prime}-\varepsilon\right||\ln | x||^{2} \exp \left\{\left|\varepsilon-\varepsilon^{\prime}\right||\ln | x||\right\}} \\
\int r_{\lambda}\left(x+y^{-1}\right) \sigma^{-1}(y) d y=0
\end{gathered}
$$

d'où l'on déduit que

$$
\lim _{\varepsilon^{\prime} \rightarrow \varepsilon}\left\|\frac{T_{\varepsilon^{\prime}}-T_{\varepsilon}}{\varepsilon^{\prime}-\varepsilon}-T_{\varepsilon}^{\prime}\right\|_{C(\overline{\mathbb{R}}, \sigma)}=0
$$

où la norme est la norme de l'espace $C(\overline{\mathbb{R}}, \sigma)$, ce qui prouve l'analyticité annoncée dans la partie d) de la Proposition VIII.1.

Enfin nous déduisons aussi facilement le point e) de la proposition : si $r$ est continue, le résultat vient directement du théorème de Lebesgue; dans le cas contraire, on approxime par des fonctions continues. 
Quant au point f) concernant $T_{\varepsilon, \lambda}^{*}$ l'adjoint de $T_{\varepsilon, \lambda}$, il se déduit des résultats précédents.

Dans la suite de cette section nous utiliserons les notations suivantes:

$$
\begin{aligned}
& \Sigma=[-2,+2]+\operatorname{Suppr}(\cdot) \\
& \stackrel{\circ}{\Sigma}=]-2,+2[+\operatorname{Suppr}(\cdot) \\
& B_{\lambda}=\text { enveloppe convexe de }\left\{\frac{1}{2}\left(\lambda-\omega+\frac{\lambda-\omega}{|\lambda-\omega|} \sqrt{(\lambda-\omega)^{2}-4} \mid \omega \in \operatorname{Supp} r(\cdot)\right\} .\right.
\end{aligned}
$$

Nous avons alors le:

Théorème VIII.3. Supposons que la densité $r$ soit dans $L_{\infty}\left((1+|x|)^{1+\alpha}\right)$ et soit $\varepsilon \in \mathbb{R}$, $|\varepsilon|<\varepsilon_{0}$ et $\lambda \in \mathbb{R}$. Alors les opérateurs $T_{\varepsilon, \lambda}$ et $T_{\varepsilon, \lambda}^{*}$ ont une valeur propre maximale $\varrho_{\varepsilon, \lambda}>0$, analytique réelle en $\varepsilon$ et continue en $\varepsilon, \lambda$. Elle est simple et excède en module les autres valeurs propres.

Les vecteurs propres $v_{\varepsilon, \lambda}$ et $\varphi_{\varepsilon, \lambda}$ associés à $\varrho_{\varepsilon, \lambda}$ sont des éléments, positifs, de $C(\overline{\mathbb{R}}, \sigma)$ et $\mathscr{M}\left(\overline{\mathbb{R}}, \sigma^{-1}\right)$, et ce sont les seuls vecteurs propres positifs de $T_{\varepsilon, \lambda}$ et $T_{\varepsilon, \lambda}^{*}$.

En conséquence nous avons les décompositions

$$
\begin{gathered}
T_{\varepsilon, \lambda} f=\varrho_{\varepsilon, \lambda} \varphi_{\varepsilon, \lambda}(f) v_{\varepsilon, \lambda}+\bar{T}_{\varepsilon, \lambda} f, f \in C(\overline{\mathbb{R}}, \sigma), \\
T_{\varepsilon, \lambda}^{*} \psi=\varrho_{\varepsilon, \lambda} \psi\left(v_{\varepsilon, \lambda}\right) \varphi_{\varepsilon, \lambda}+\bar{T}_{\varepsilon, \lambda}^{*} \psi, \psi \in \mathscr{M}\left(\overline{\mathbb{R}}, \sigma^{-1}\right),
\end{gathered}
$$

où les rayons spectraux de $\bar{T}_{\varepsilon, \lambda}$ et $\bar{T}_{\varepsilon, \lambda}^{*}$ sont strictement inférieurs à $\varrho_{\varepsilon, \lambda}$ et continus en $\varepsilon$ et $\lambda$. On peut de plus, choisir $v_{\varepsilon, \lambda}$ et $\varphi_{\varepsilon, \lambda}$ de sorte que

$$
\int v_{\varepsilon, \lambda}(x) d x=1 \text { et } \int \varphi_{\varepsilon, \lambda}(d x) v_{\varepsilon, \lambda}(x)=1 .
$$

De plus, $\varphi_{\varepsilon, \lambda}$ est une mesure strictement positive sur $\mathbb{R}$. Lorsque $\lambda \in \Sigma^{\circ}, v_{\varepsilon, \lambda}$ est aussi strictement positif sur $\mathbb{R}$. Lorsque $\lambda \in \mathbb{R} \backslash \stackrel{\circ}{\Sigma}$, le support de $v_{\varepsilon, \lambda}$ contient $B_{\lambda}$.

Preuve. Nous présentons d'abord un lemme technique de positivité qui sera utile dans la preuve; un résultat semblable aux parties b) et c) de ce lemme a été utilisé déjà par O’Connor [21].

Lemme VIII.4. Soit $T_{\varepsilon, \lambda}^{n}(x \mid y)$ le noyau de $T_{\varepsilon, \lambda}^{n}$. Alors

a) $\forall y, T_{\varepsilon, \lambda}^{(n)}(x \mid y)$ est continu en $x$ pour $n \geqq 2$.

b) $\forall \lambda \in \sum$ et $\forall x \in \mathbb{R}, \exists Y \subset \mathbb{R}, Y$ dense dans $\mathbb{R}$ tel que $\forall y \in Y, T_{\varepsilon, \lambda}^{(n)}(x \mid y)>0$ pour $n$ assez grand.

c) $\forall \lambda \in \sum^{\circ}$ et $\forall x \in \mathbb{R}, \forall \mathscr{K}$ compact de $\mathbb{R}, T_{\varepsilon, \lambda}^{(n)}(x \mid y)>0, \forall y \in \mathscr{K}$ pour $n$ assez grand.

d) $\forall \lambda \in \mathbb{R} \backslash \sum^{\circ}, \forall \mathscr{K}$ compact de $B_{\lambda}, \forall y \in \mathbb{R}, T_{\varepsilon, \lambda}^{(n)}(x \mid y)>0, \forall x \in \mathscr{K}$ pour $n$ assez grand.

Preuve. La partie a) découle directement des techniques utilisées dans la preuve de la Proposition VIII.1.

La partie b) se déduit essentiellement du fait que la transformation $y \rightarrow x=\lambda$ $-a-y^{-1}$ peut s'écrire sous la forme

$$
\frac{x-z_{0}}{x-z_{0}^{\prime}}=\frac{z_{0}^{\prime}}{z_{0}} \frac{y-z_{0}}{y-z_{0}^{\prime}}
$$

où $z_{0}$ et $z_{0}^{\prime}$ en désignent les points fixes et de ce que si $\lambda \in \stackrel{\circ}{\Sigma}$, on peut toujours trouver $a \in \operatorname{Supp} r(\cdot)$ tel que ces points fixes soient de module 1 et que $\frac{1}{2 \pi} \operatorname{Arg}\left(\frac{z_{0}^{\prime}}{z_{0}}\right)$
soit irrationnel. 
La partie c) est une conséquence de la partie b) et de la continuité.

La partie d) se déduit par la continuité en utilisant que $B_{\lambda}$ est précisément l'enveloppe convexe de l'ensemble des points fixes attracteurs de la transformation

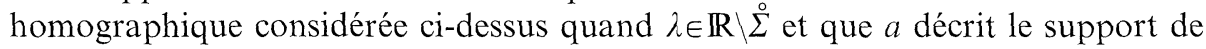
$r(\cdot)$.

Revenons donc maintenant à la preuve du Théorème VIII.3. Commençons par étudier le cas où $\lambda \in \stackrel{\circ}{\Sigma}$. De la propriété de positivité prouvée dans le lemme cidessus, il suit ([15], Théorème 6.2) l'existence d'une valeur propre $\varrho_{\varepsilon, \lambda}$ positive et maximale de $T_{\varepsilon, \lambda}$ et de $T_{\varepsilon, \lambda}^{*}$ et des vecteurs propres correspondants $v_{\varepsilon, \lambda}$ et $\varphi_{\varepsilon, \lambda}$, éléments positifs de $C(\overline{\mathbb{R}}, \sigma)$ et $\mathscr{M}\left(\overline{\mathbb{R}}, \sigma^{-1}\right)$. Leur stricte positivité est aussi une conséquence du lemme ci-dessus.

Nous voulons maintenant montrer que $\varrho_{\varepsilon, \lambda}$ est, de plus, une valeur propre simple et qu'elle excède en module les autres valeurs propres. Nous utiliserons des techniques classiques, parallèles à la preuve de la proposition $\left(\beta^{\prime}\right)$ de la référence [15]. Nous supprimerons les indices $\varepsilon$ et $\lambda$, et nous écrirons simplement $\varrho, T, v, \varphi$. Supposons qu'il existe une valeur propre $\mu$ de même module que $\varrho, \mu=\varrho \exp \{i \theta\}$, de vecteur propre $w, \theta$ pouvant être nul. Alors $|w|$ est vecteur propre de $T$ associé à la valeur propre $\varrho=|\mu|$ et de plus $|w(x)|>0, \forall x \in \mathbb{R}$. En effet

$$
\varphi(T|w|-\varrho|w|)=0
$$

or $T|w| \geqq \varrho(|w|)$ et $\varphi$ positive, donc $T|w|=\varrho|w|$. La positivité vient de $|w|=\varrho^{-n} T^{n}|w|$ et du Lemme VIII.4. Montrons alors maintenant que $w=|w| \exp \{i \alpha\}$ où $\alpha$ est une phase constante:

$$
\begin{aligned}
\int T^{(n)}(x \mid y)|w(y)| d y & =\varrho^{n}|w(y)|=\left|\varrho^{n} e^{i n_{\theta}} w(y)\right| \\
& =\left|\int T^{(n)}(x \mid y) w(y) d y\right|
\end{aligned}
$$

où $T^{(n)}(x \mid y)$ dénote le noyau de $T^{n}$. Il s'en déduit qu'il existe $\alpha(n)$, tel que $\forall x$ :

$$
T^{(n)}(x \mid y) w(y)=\exp \{i \alpha(n)\} T^{(n)}(x \mid y)|w(y)|
$$

pour presque tout $y$, soit encore

$$
w(y)=\exp \{i \alpha(n)\}|w(y)|
$$

pour presque tout $y$ tel que $T^{(n)}(x \mid y)>0$ pour un certain $x$. Utilisant de nouveau le lemme de positivité VIII.4, on conclut que $w=|w| \exp \{i \alpha\}$ pour tout $y \in \mathbb{R}$.

Il ne reste plus à montrer que $T$ ne peut avoir deux vecteurs propres $v, w$ strictement positifs sur $\mathbb{R}$, associés à la valeur propre $\varrho$. Or cela impliquerait que $\varphi(\alpha v+\beta w)=0$ pour $\alpha$ et $\beta$ appropriés. Mais $\varphi$ étant positive sur $\mathbb{R}$, cela implique que $\alpha v+\beta w$ changerait de signe, ce qui est contradictoire avec le résultat ci-dessus, que la phase est la même presque partout. c.q.f.d.

Un argument simple assure alors qu'il ne peut y avoir d'autres vecteurs propres positifs de $T$ et $T^{*}$ correspondant à des valeurs propres différentes de $\varrho$.

L'analyticité et la continuité de $\varrho$ résultent de celles de $T$ et de ce que $\varrho$ est simple.

Les décompositions (VIII.10) et (VIII.11) de $T$ et $T^{*}$ et la propriété des rayons spectraux de $\bar{T}$ et $\bar{T}^{*}$ est une conséquence immédiate de ce que la valeur propre $\varrho$ est non dégénérée et domine les autres valeurs propres en module. 
Lorsque $\lambda \in \mathbb{R} \backslash \stackrel{\circ}{\Sigma}$, on commence par constater que $T_{\varepsilon, \lambda}$ et $T_{\varepsilon, \lambda}^{*}$ ont une valeur propre maximale $\varrho_{\varepsilon, \lambda}$ dont les vecteurs propres $v_{\varepsilon, \lambda}$ et $\varphi_{\varepsilon, \lambda}$ sont positifs. Utilisant les propriétés d) du Lemme VIII.4, on vérifie que $\varphi_{\varepsilon, \lambda}$ est un élément strictement positif sur $\mathbb{R}$ de $\mathscr{M}\left(\overline{\mathbb{R}}, \sigma^{-1}\right)$ et que $v_{\varepsilon, \lambda}$ contient $B_{\lambda}$ dans son support. La preuve est dès lors proche de celle du cas où $\grave{\lambda} \in \dot{\Sigma}$.

Nous avons besoin de renseignements précis sur la valeur propre maximale $\varrho_{\varepsilon, \lambda}$ de $T_{\varepsilon, \lambda}$ et $T_{\varepsilon, \lambda}^{*}$.

Théorème VIII.5. Supposons que la densité $r(\cdot)$ soit dans $L_{\infty}\left((1+|x|)^{1+\alpha}\right)$ et soit $\varepsilon \in \mathbb{R}$, $|\varepsilon|<\varepsilon_{0}$. La valeur propre maximale $\varrho_{\varepsilon, \lambda}$ de $T_{\varepsilon, \lambda}$ et de $T_{\varepsilon, \lambda}^{*}$ satisfait les propriétés suivantes:

a) $\varrho_{0, \lambda}=1$ et $\left.\frac{d}{d \varepsilon} \varrho_{\varepsilon, \lambda}\right|_{\varepsilon=0}=-\int v_{0, \lambda}(x) \ln |x| d x<0$.

b) Posons $\left.\frac{d}{d \varepsilon} \varrho_{\varepsilon, \lambda}\right|_{\varepsilon=0}=-\frac{\gamma(\lambda)}{2}, \gamma(\lambda)>0$.

Alors $\gamma(\lambda)$ est continu en $\lambda$ et

$$
\ln \varrho_{\varepsilon, \lambda} \geqq-\varepsilon \frac{\gamma(\lambda)}{2} .
$$

c) Pour tout compact $\mathscr{K}$ de $\mathbb{R}$, il existe $\varepsilon_{1}(\mathscr{K}), 0<\varepsilon_{1}<\varepsilon_{0}$ tel que

$$
\ln \varrho_{\varepsilon, \lambda} \leqq-\varepsilon \frac{\gamma(\lambda)}{4}
$$

quand $\lambda \in \mathscr{K}$ et $0 \leqq \varepsilon \leqq \varepsilon_{1}(\mathscr{K})$.

d) Pour tout $\varepsilon, 0<\varepsilon<\varepsilon_{0}$, il existe $\lambda_{0}(\varepsilon)$ tel que

pour $\lambda>\lambda_{0}(\varepsilon)$.

$$
\varrho_{\varepsilon, \lambda} \leqq \lambda^{-\varepsilon / 2}
$$

Remarque. On peut en fait, montrer que $\gamma(\lambda)$ est l'exposant de Lyapounov du produit de matrices aléatoires $2 \times 2$ associé à l'équation de Schrödinger unidimensionnelle discrétisée avec un potentiel eléatoire. Que $\gamma(\lambda)$ soit positif est alors une conséquence du théorème de Furstenberg [9] montrant la croissance exponentielle de la norme de tels produits (Matsuda et Ishii [17], puis Yosioka [33] ont vérifié que ce théorème s'applique dans ce cas). Nous donnons dans le cas particulier qui nous intéresse une nouvelle preuve de ce résultat, naturelle dans notre contexte.

Prewve. Que 1 soit valeur propre de $T_{0, \lambda}$ et $T_{0, \lambda}^{*}$ ressort directement du fait que la mesure de Lebesgue sur $\mathbb{R}, 1 \times d x$ qui appartient à $\mathscr{M}\left(\mathbb{R}, \sigma^{-1}\right)$ est vecteur propre de $T_{0, \lambda}^{*}$. La valeur propre 1 est alors nécessairement la valeur propre maximale $\varrho_{0, \lambda}$ $\operatorname{car} \varphi_{0, \lambda}$ est le seul vecteur propre dans le cône positif (Théorème VIII.3).

L'égalité dans (VIII.17) s'obtient en dérivant l'identité

$$
\varrho_{\varepsilon, \lambda} \varphi_{\varepsilon, \lambda}\left(v_{\varepsilon, \lambda}\right) \equiv \iint|x|^{-\varepsilon} r_{\lambda}\left(x+y^{-1}\right) \varphi_{\varepsilon, \lambda}(d x) v_{\varepsilon, \lambda}(y) d y
$$

et en utilisant le fait que $\varphi_{\varepsilon, \lambda}\left(v_{\varepsilon, \lambda}\right)=1$ pour petit $\varepsilon$ et que $\varphi_{0, \lambda}(d x)=1 \times d x$, la permutation de l'intégration et de la dérivation étant légitimée par les propriétés 
d'intégrabilité de $\varphi_{\varepsilon, \lambda}$ et de $v_{\varepsilon, \lambda}$. L'inégalité dans (VIII.17) est obtenue de la façon suivante:

$$
\begin{aligned}
\left.\frac{d}{d \varepsilon} \varrho_{\varepsilon, \lambda}\right|_{\varepsilon=0} & =\frac{1}{2} \int v_{0, \lambda}(x) \ln |x|^{-2} d x \\
& =\frac{1}{2} \iint v_{0, \lambda}(x) \ln \left[|x|^{-2} \frac{v_{0, \lambda}(y)}{v_{0, \lambda}(x)}\right] r_{\lambda}\left(y+x^{-1}\right) d x d y \\
& \leqq \frac{1}{2} \iint v_{0, \lambda}(x) r_{\lambda}\left(y+x^{-1}\right)\left\{|x|^{-2} \frac{v_{0, \lambda}(y)}{v_{0, \lambda}(x)}-1\right\} d x d y=0
\end{aligned}
$$

et de plus l'inégalité (VIII.22) est stricte sauf si

$$
\left.\iint|| x\right|^{-2} v(y)-v(x) \mid r_{\lambda}\left(y+x^{-1}\right) d x d y=0
$$

soit encore

$$
\left.\int_{-\infty}^{+\infty}|| x\right|^{-2} v\left(\xi-x^{-1}\right)-v(x) \mid d x=0
$$

pour $r_{\lambda}(\cdot)$ presque tout $\xi$. Or nous avons

$$
\begin{aligned}
\left.\int_{-\infty}^{+\infty}|| x\right|^{-2} v\left(\xi-x^{-1}\right)-v(x) \mid d x & \geqq\left|\int_{-\infty}^{a}\left\{|x|^{-2} v\left(\xi-x^{-1}\right)-v(x)\right\} d x\right| \\
& =\left|\int_{0}^{1 / a} v(\xi-y) d y-\int_{-\infty}^{a} v(x) d x\right|
\end{aligned}
$$

qui est strictement positif pour $a$ assez grand. Ceci achève la preuve de la partie a) du théorème, en mentionnant cependant que les égalités et inégalités (VIII.20) à (VIII.22) sont justifiées par le fait que $v_{0, \lambda}$ est un élément du cône positif de $C(\overline{\mathbb{R}}, \sigma)$; lorsque le support de $\nu_{0, \lambda}$ n'est pas $\mathbb{R}$ tout entier, les intégrations sont restreintes au support de $v_{0, \lambda}$.

Prouvons maintenant la partie b) du théorème. La continuité de $\gamma(\lambda)$ découle de la formule (VIII.17) et de ce que le vecteur propre $v_{0, \lambda}$ est continu en $\lambda$. La borne inférieure sur $\ln \varrho_{\varepsilon, \lambda}$ se déduit de ce que $\ln \varrho_{\varepsilon, \lambda}$ est une fonction convexe de $\varepsilon$ pour petit $\varepsilon$. En fait, plus généralement si $R_{\varepsilon, \lambda}$ est le rayon spectral de $T_{\varepsilon, \lambda}$ (qui pour petit $\varepsilon$ coïncide avec $\varrho_{\varepsilon, \lambda}$ gràce au Théorème VIII.3) alors $\ln R_{\varepsilon, \lambda}$ est convexe en $\varepsilon$ pour tout $\varepsilon$ gràce à l'inégalité de Schwarz:

$$
\begin{aligned}
& \ln R_{\left(\varepsilon_{1}+\varepsilon_{2}\right) / 2, \lambda}=\lim _{n \rightarrow \infty} \frac{1}{n} \ln \left\|T_{\left(\varepsilon_{1}+\varepsilon_{2}\right) / 2, \lambda}^{n}\right\| \leqq \lim _{n \rightarrow \infty} \frac{1}{n} \\
& \cdot \ln \left|\operatorname{Sup}_{\substack{\psi \in C(\overline{\mathbb{R}}, \sigma) \\
\| \psi \mid=1 \\
x_{n} \in \mathbb{R}}} \sigma\left(x_{n}\right) \int d x_{n-1} \ldots d x_{0}\left(\left|x_{n}\right| \ldots\left|x_{1}\right|\right)^{-\left(\varepsilon_{1}+\varepsilon_{2}\right) / 2} r_{\lambda}^{(n)}\left(x_{n}, \ldots, x_{0}\right) \psi\left(x_{0}\right)\right| \\
& \leqq_{\overline{\mathbb{R}}} \lim _{n \rightarrow \infty} \frac{1}{n} \ln \operatorname{Sup}_{\substack{\psi \in C(\mathbb{R}, \sigma) \\
\| \psi(x)=1}}\left\{\sigma\left(x_{n}\right) \int d x_{n-1} \ldots d x_{0}\left|x_{n} \ldots x_{1}\right|^{-\varepsilon_{1}} r_{\lambda}^{(n)}\left(x_{n} \ldots x_{1}\right)\right. \\
& \left.\cdot\left|\psi\left(x_{1}\right)\right| \cdot \sigma\left(x_{n}\right) \int d x_{n-1} \ldots d x_{0}\left|x_{n} \ldots x_{1}\right|^{-\varepsilon_{2}} r_{\lambda}^{(n)}\left(x_{n} \ldots x_{0}\right)\left|\psi\left(x_{0}\right)\right|\right\}^{1 / 2} \\
& \leqq \frac{1}{2}\left(\ln R_{\varepsilon_{1}, \lambda}+\ln R_{\varepsilon_{2}, \lambda}\right)
\end{aligned}
$$

où $r_{\lambda}^{(n)}\left(x_{n}, \ldots, x_{0}\right)$ désignait le produit de facteurs $r_{\lambda}\left(x_{k+1}+x_{k}^{-1}\right)$ associé à $\mathrm{T}_{0, \lambda}^{n}$. 
L'inégalité (VIII.18) (la partie c) du théorème est obtenue par un développement de Taylor de $\ln \varrho_{\varepsilon, \lambda}$ au second ordre en $\varepsilon$ qui est possible dans un voisinage de $\varepsilon=0$, puisque $\varrho_{0, \lambda}=1$. La dérivée seconde est bornée uniformément en utilisant une formule de Cauchy exploitant l'analyticité de $\varrho_{\varepsilon, \lambda}$ en $\varepsilon$ au voisinage de 0 et la continuité jointe en $\varepsilon$ et $\lambda$ de $\varrho_{\varepsilon} \lambda$.

Enfin la partie d) du théorème s'obtient en montrant que $\varrho_{\varepsilon, \lambda}$ satisfait une inégalité de la forme

$$
\varrho_{\varepsilon, \lambda} \leqq a_{\varepsilon}(\lambda)+b_{\varepsilon}(\lambda) \varrho_{\varepsilon, \lambda}^{-1}
$$

où $a_{\varepsilon}(\lambda)$ et $b_{\varepsilon}(\lambda)$ se comportent de façon appropriée lorsque $\lambda$ tend vers l'infini. Cette inégalité est obtenue en jouant sur l'identité

$$
\varrho_{\varepsilon, \lambda^{v}} \varepsilon_{\varepsilon, \lambda}(x)=|x|^{-\varepsilon} \int r_{\lambda}\left(x+y^{-1}\right) v_{\varepsilon, \lambda}(y) d y .
$$

Nous indiquons maintenant une conséquence essentielle de ces résultats

Corollaire VIII.6. Soit $\mathscr{K}$ un compact de $\mathbb{R}$. Alors

$$
\left|\int \psi(d x)\left(T_{\varepsilon, \lambda}^{n} f\right)(x)\right| \leqq C \exp \left\{-\varepsilon \frac{\gamma(\hat{\lambda})}{4} n\right\}\|f \sigma\|_{\infty}\left\|\psi \sigma^{-1}\right\|_{1}
$$

pour tout $\lambda \in \mathscr{K}$ et $\varepsilon, 0 \leqq \varepsilon<\varepsilon_{1}(\mathscr{K})$, et pour tout $f \in B(\mathbb{R}, \sigma)$ et $\psi \in \mathscr{M}\left(\overline{\mathbb{R}}, \sigma^{-1}\right)$. C ne dépend que de $\mathscr{K}$ et $\varepsilon$.

Preuve. La borne (VIII.27) découle directement de ce que $T_{\varepsilon, \lambda}$ envoie $B(\mathbb{R}, \sigma)$ dans $C(\overline{\mathbb{R}}, \sigma)$ et que d'après le Théorème VIII.3

$$
T_{\varepsilon, \lambda}=\varrho_{\varepsilon, \lambda} \varphi_{\varepsilon, \lambda}(\cdot) v_{\varepsilon, \lambda}+\bar{T}_{\varepsilon, \lambda}
$$

et donc

$$
T_{\varepsilon, \lambda}^{p}=\varrho_{\varepsilon, \lambda}^{p} \varphi_{\varepsilon, \lambda}(\cdot) v_{\varepsilon, \lambda}+\bar{T}_{\varepsilon, \lambda}^{p}
$$

d'où

$$
\left\|T_{\varepsilon, \lambda}^{p}\right\|_{C_{(\overline{\mathbb{R}}, \sigma)}} \leqq D(\varepsilon) \varrho_{\varepsilon, \lambda}^{p}+\left\|\bar{T}_{\varepsilon, \lambda}^{p}\right\|_{\mathcal{C}(\overline{\mathbb{R}}, \sigma)}
$$

avec

$$
D(\varepsilon)=\left\|\varphi_{\varepsilon, \lambda}\right\|_{\mathcal{M}\left(\overline{\mathbb{R}}, \sigma^{-1}\right)}\left\|v_{\varepsilon, \lambda}\right\|_{C(\overline{\mathbb{R}}, \sigma)}
$$

Mais le rayon spectral de $\bar{T}_{\varepsilon, \lambda}$ est uniformément plus petit que $\varrho_{\varepsilon, \lambda}$ dans le domaine considéré en $\varepsilon$ et $\lambda$.

Corollaire VIII.7. Pour tout compact $\mathscr{K}$ de $\mathbb{R}$, il existe $\chi>0$ tel que $\forall \lambda \in \mathscr{K}$ et $\forall f \in B(\mathbb{R}, \sigma)$

$$
\left|\left(\int f(y) d y\right) v_{0, \lambda}(x)-\left(T_{0, \lambda}^{n} f\right)(x)\right| \leqq \sigma^{-1}(x)\|f \cdot \sigma\|_{\infty} \cdot \exp \{-\chi n\} .
$$

Preuve. Ce résultat de convergence exponentielle se déduit directement de la décomposition (VIII.10).

\section{Résultats sur l'opérateur de Schrödinger unidimensionnel}

Nous utilisons maintenant les résultats obtenus dans les sections précédentes pour déduire des renseignements sur la décroissance des corrélations, la nature du 
spectre et des fonctions propres, la conductivité électrique statique associées à l'opérateur de Schrödinger discrétisé unidimensionnel. Nous obtiendrons aussi diverses formules explicites pour des quantités d'intérêt.

Dans toute cette section, comme dans la précédente, nous supposons que la densité de probabilité $r(\cdot)$ appartient à $L_{\infty}\left((1+|x|)^{1+\alpha}\right)$ pour un certain $\alpha$, $0<\alpha<1$ ainsi que $\beta(\cdot)$ et $\delta(\cdot)$.

Nous énonçons

Théorème IX.1. Soit $H(\omega)$ l'opérateur de Schrödinger unidimensionnel défini par

$$
(H \psi)(x)=-\psi(x+1)-\psi(x-1)+\omega_{x} \psi(x)
$$

où $x \in \mathbb{Z}$, ou bien $x \in \mathbb{N}$ et $\psi(-1)=0$.

Les $\omega_{x}$ sont des variables aléatoires indépendantes de même loi $r(\cdot)$, la distribution produit étant notée $\mu$. Alors avec probabilité 1, le spectre de $H(\omega)$ est $\Sigma=[-2,+2]+\operatorname{supp} r(\cdot)$. De plus,

a) Les fonctions de corrélation associées satisfont

$$
\left|\varrho_{\mu}(x, y ; A)\right| \leqq \varrho_{\mu}(x, y ; A) \leqq D \cdot \exp \{-\chi(A)|x-y|\}
$$

pour tout intervalle $A$ borné de $\mathbb{R}$, et $D$ ne dépend que de $A$.

Les mêmes bornes sont satisfaites uniformément en $\Lambda$ pour les corrélations à volume fini $\varrho_{\mu}^{\Lambda}(x, y ; A)$ et $\varrho_{\mu}^{\Lambda}(x, y ; A)$.

b) Avec probabilité 1, le spectre de $H(\omega)$ est purement ponctuel et les fonctions propres décroissent exponentiellement.

c) Avec probabilité 1, la constante de diffusion $D(A)$ est nulle, et la conductivité électrique statique $\sigma_{H}$, associée à $H(\omega)$ s'annule lorsque la température tend vers zéro. De plus si le support de $r(\cdot)$ est borné, la conductivité électrique statique $\sigma_{H}$ est nulle pour toute température et la conductivité en fréquence $\sigma_{H}(\omega)$ satisfait

$$
\liminf _{\Lambda \uparrow S} \frac{1}{\omega} \int_{0}^{\omega_{0}} \operatorname{Re} \sigma_{H}^{A}(\omega)=0\left(\omega_{0}\right) .
$$

Preuve. Le résultat sur le spectre $\Sigma$ suit du Théorème III.2. La partie a) du théorème découle de la Proposition VII.3 et du Corollaire VIII.6. La partie b) découle de la partie a) grâce au Corollaire IV.5, en remarquant de plus pour obtenir la décroissance des fonctions propres que les valeurs propres ne sont jamais dégénérées (ceci se vérifie facilement par un analogue de la preuve classique de ce que les valeurs propres de l'opérateur de Schrödinger continu sont de multiplicité 1). Enfin, la partie c) suit de la partie a) par les Corollaires V.3 et V.5 et le Théorème V.4.

Remarque. Les bornes obtenues sur les fonctions de corrélation dans la partie a) sont indépendantes des densités de probabilité $\beta(\cdot)$ et $\delta(\cdot)$ choisies à la frontière de $\Lambda$, et les résultats b) et c) en sont donc aussi indépendants.

Pour les formules explicites suivantes, nous supposerons par simplicité le cas de la droite entière $\mathbb{Z}$ et non pas le cas de la demi-droite $\mathbb{N}$. Des formules explicites s'obtiennent cependant aussi facilement dans ce dernier cas. 
Théorème IX.2. Les formules explicites suivantes sont vérifiées, $\forall x$ et $x^{\prime} \in \mathbb{Z}, x \leqq x^{\prime}$ et $\forall A$ intervalle ouvert borné de $\mathbb{R}$ :

a) la mesure spectrale moyenne est donnée par

$$
\begin{aligned}
& \lim _{\Lambda \uparrow \mathbb{Z}} \varrho^{\Lambda}\left(x, x^{\prime} ; A\right)=\varrho\left(x, x^{\prime} ; A\right) \\
& \quad=\frac{1}{2} \int_{A} d \lambda \int_{\mathbb{R}}\left(\stackrel{\circ}{T}_{1, \lambda}^{x^{\prime}-x} v_{0, \lambda}\right)(y) v_{0, \lambda}\left(y^{-1}\right) d y
\end{aligned}
$$

et en particulier la densité d'états, intégrée dans $A$ est donnée par

$$
\varrho(0,0 ; A)=\frac{1}{2} \int_{A} d \lambda v_{0, \lambda}(y) v_{0, \lambda}\left(y^{-1}\right) d y
$$

b) la fonction de corrélation entre $x$ et $x^{\prime}$ satisfait

$$
\begin{aligned}
\bar{\varrho}\left(x, x^{\prime} ; A\right) & \leqq \lim _{\Lambda \uparrow \mathbb{Z}} \bar{\varrho}^{\Lambda}\left(x, x^{\prime} ; A\right) \\
& =\frac{1}{2} \int_{A} d \lambda \int_{\mathbb{R}}\left(T_{1, \lambda}^{x^{\prime}-x} v_{0, \lambda}\right)(y) v_{0, \lambda}\left(y^{-1}\right) d y
\end{aligned}
$$

où les opérateurs $\stackrel{\circ}{T}_{1, \lambda}$ et $T_{1, \lambda}$ ont été définis dans la Sect. VII et où $v_{0, \lambda}$ est la seule solution continue, positive sur $\mathbb{R}$ de l'équation intégrale

satisfaisant $\int v_{0, \lambda}(x) d x=1$.

$$
v_{0, \lambda}(x)=\int r\left(\lambda-x-y^{-1}\right) v_{0, \lambda}(y) d y
$$

c) la mesure de transfert entre $x$ et $x^{\prime}$ satisfait:

$$
\begin{aligned}
R(x, y ; A) \geqq & \lim _{\Lambda \uparrow S} R^{\Lambda}(x, y ; A) \\
= & \int_{A} d \lambda \int_{\mathbb{R}^{y-x+3}} \frac{\psi^{2}(0) \psi(y-x)^{2}}{|\psi(-1) \psi(y-x+1)|} \\
& \cdot a(\psi(y-x), \psi(y-x+1)) a(\psi(0), \psi(-1)) \\
& \cdot \prod_{0 \leqq z \leqq y-x} r_{\lambda}\left(\frac{\psi(z-1)+\psi(z+1)}{\psi(z)}\right) \\
& \cdot \prod_{-1 \leqq z \leqq y-x+1}\left\{e^{-\psi(z)^{2}} \frac{d \psi(z)}{|\psi(z)|}\right\}
\end{aligned}
$$

où $a(s, t)$ est solution positive de l'équation intégrale

$$
a(s, t)=\int r_{\lambda}\left(s t^{-1}+y_{n}^{-1}\right) e^{-t^{2}} a\left(t, t y_{n}^{-2}\right) d y_{n} .
$$

Preuve. Les résultats des parties a) et b) du Théorème IX.2 sont des conséquences directes des formules établies pour les corrélations à volume fini (Proposition VII.2) et des résultats prouvés sur l'opérateur de transfert dans la Sect. VIII. La partie c) du théorème se déduit quant à elle des formules explicites de la Sect. VII pour la mesure de récurrence à volume fini (Proposition VII.4) à travers l'argument suivant: partant de la formule (VII.16) nous voyons que $a_{n}(s, t \mid f)$ satisfait la relation de récurrence

$$
\begin{aligned}
a_{n}(s, t \mid f)=\int & T_{0, \lambda}\left(s t^{-1} \mid y_{n}\right) e^{-t^{2}} \\
& \cdot a_{n-1}\left(t, t y_{n}^{-2} \mid f\right) d y_{n}
\end{aligned}
$$


où l'opérateur $T_{0, \lambda}$ a été défini dans la Sect. VII. Considérant son vecteur propre $v_{0, \lambda}$ associé à la valeur propre maximale 1 (Théorèmes VIII.3 et VIII.5) nous voyons que

$$
0 \leqq a_{n}\left(s, t \mid v_{0, \lambda}\right) \leqq a_{n-1}\left(s, t \mid v_{0, \lambda}\right) \leqq v_{0, \lambda}(s / t)
$$

doù il suit que $\lim _{n \rightarrow \infty} a_{n}\left(s, t v_{v_{0, \lambda}}\right)=a(s, t)$ existe, et satisfait l'équation (IX.9). Il ne suffit alors plus que de montrer que $\forall f \in L_{\infty}\left((1+|x|)^{1+\alpha}\right), \lim _{n \rightarrow \infty} a_{n}(s, t \mid f)=a(s, t)$. Cette dernière étape est accomplie de la façon suivante; on écrit

$$
a_{n}(s, t \mid f)=a_{n}^{\prime}(s, t \mid f)+a_{n}^{\prime \prime}(s, t \mid f)
$$

où $a_{n}^{\prime}$ et $a_{n}^{\prime \prime}$ sont obtenus en remplacant dans l'expression (VII.16) de $a_{n}$ le facteur

$$
\exp \left\{-\psi(1)^{2} \sum_{1 \leqq z \leqq n}\left|y_{n} \ldots y_{n-z+1}\right|^{2}\right\}
$$

respectivement par

et par

$$
\exp \left\{-\psi(1)^{2} \sum_{[n / 2] \leqq z \leqq n}\left|y_{n} \ldots y_{n-z+1}\right|^{2}\right\}
$$

$$
\exp \left\{-\psi^{2}(1) \sum_{1 \leqq z \leqq n}\left|y_{n} \ldots y_{n-z+1}\right|^{2}\right\}-\exp \left\{-\psi(1)^{2} \sum_{[n / 2] \leqq z \leqq n}\left|y_{n} \ldots y_{n-z+1}\right|^{2}\right\},
$$

où $[n / 2]$ désigne la partie entière de $n / 2$. On constate alors que

$$
\lim _{n \rightarrow \infty} a_{n}^{\prime}(s, t \mid f)=a(s, t)
$$

parce que $\lim _{n \rightarrow \infty} T_{0, \lambda}^{[n / 2]} f=v_{0, \lambda}$. D'autre part, utilisant l'inégalité $1-\exp \left\{-\sum_{j=1}^{n} x_{j}\right\}$ $\leqq \sum_{j=1}^{n} x_{j}^{\varepsilon}, x_{j} \geqq 0,0 \leqq \varepsilon \leqq 1$, nous pouvons déduire que $a_{n}^{\prime \prime}(s, t \mid f)$ tend exponentiellement vite vers 0 avec $n$ en choisissant $\varepsilon$ assez petit et utilisant le Corollaire VIII.6. Le théorème de la convergence dominée achève alors la preuve.

Remarques. Les limites établies dans le théorème ci-dessus sont donc indépendantes des distributions $\beta$ et $\delta$ choisies aux bords de la boîte. On vérifie facilement de plus que les limites sont atteintes exponentiellement vite, grâce, au Corollaire VIII.7.

\section{La transition de localisation vue comme un changement de phase: description qualitative}

Un des aspects de notre travail a été de donner une base solide à l'analogie entre le problème de la localisation et celui des transitions de phase.

Nous avons montré qu'il était possible de représenter diverses valeurs moyennes de fonctions associées aux équations aléatoires par des intégrales multiples qui peuvent s'interpréter comme des fonctions de corrélation d'un système de spins continus sur un réseau. Les divers critères spectraux que nous 
avons obtenus nous permettent d'établir un lien entre la nature du spectre de l'hamiltonien et certaines propriétés qualitatives de ces fonctions de corrélation. C'est le cas notamment de la fonction de corrélation $\bar{\varrho}(x, y)$ qui mesure la corrélation entre les valeurs absolues d'une fonction propre au point $x$ et au point $y$. Nous avons vu que lorsque cette fonction décroit de façon suffisamment rapide, tous les états sont localisés, c'est-à-dire que le spectre est purement ponctuel, et le système est un isolant. Ceci nous amène naturellement à penser que les états localisés jouent dans notre problème le rôle de la phase gazeuse ou paramagnétique en mécanique statistique. Lorsque le spectre de l'hamiltonien est absolument continu, cette fonction $\varrho(x, y)$ ne s'annule probablement pas lorsque les points $x$ et $y$ sont très éloignés. Elle montre alors la présence d'un ordre à grande distance dans le système. Ceci nous amène à penser que les états étendus correspondent à une phase ordonnée, analogue à celle d'un ferroaimant isotrope à basse température, qui est stable en dimension trois. Une nouvelle situation est concevable, à savoir que cette fonction tend vers 0 , c'est-à-dire ne montre pas d'ordre à grande distance, mais très lentement, de façon à ne pas être intégrable. Ceci pourrait correspondre à la présence de spectre singulier continu. Cette situation pourrait se rencontrer dans le cas bidimensionnel, et constituerait l'analogue de la phase topologiquement ordonnée du modèle $X-Y$, analysée par Kosterlitz et Thouless.

A l'heure actuelle, il ne s'agit là que de conjectures qui demandent à être étayées. Notons toutefois que l'analyse du cas unidimensionnel que nous avons faite les confirme pleinement, puisque le système n'est alors que dans une seule «phase»: tous les états sont exponentiellement localisés. Les techniques mêmes utilisées dans notre approche (opérateur de transfert) correspondent étroitement à celles utilisées en mécanique statistique des systèmes unidimensionnels.

Une étude étayée des dimensions deux et trois devrait permettre de trancher en faveur des idées développées ci-dessus ou de les rejeter.

Remerciements. Durant le cours de ce travail, nous avons bénéficié d'utiles discussions à Paris avec G. Gallavotti, F. Ledrappier, G. Royer, L. Schwartz et tout particulièrement avec M. Duneau et P. Renouard. Nous les en remercions très vivement.

H. Kunz remercie aussi N. Kuiper et J. Lascoux pour leur hospitalité lors de séjours à l'I.H.E.S. et au C.P.T. de l'Ecole Polytechnique et B. Souillard remercie Ph. Choquard et l'E.P.F.L. pour leur hospitalité à Lausanne.

\section{Bibliographie}

1. Amrein, W.O., Georgescu, V.: Helv. Phys. Acta 46, 635 (1973)

2. Anderson, P.W.: Phys. Rev. 109, 1492 (1958)

3. Anderson, P.W.: Rev. Mod. Phys. 50, 191 (1978)

4. Berge, C.: Graphes et Hypergraphes. Paris: Dunod 1970

5. Borland, R.E.: Proc. R. Soc. London. Ser. A. 274, 529 (1963)

6. Bourbaki, N.: Eléments de mathématiques-Intégration. Paris: Hermann 1960

7. Casher, A., Lebowitz, J.L.: J. Math. Phys. N.Y. 12, 1701 (1971)

8. Eggarter, T.P.: J. Math. Phys. N.Y. 14, 1308 (1973)

9. Furstenberg, H.: Am. Math. Soc. 108, 337 (1963)

10. Gol'dsheid, I.Ya.: Communication au Symposium International de Théorie de l'Information. Tbilissi, Juillet (1979)

11. Gol'dsche1d, I.Ya., Molchanov, S.A., Pastur, L.A. : Funkts. Anal. Prilozhen 11, 1 (1977) 
12. Halperin, B.I.: Adv. Chem. Phys. 13, 123 (1967)

13. Ishii, K.: Supp. Prog. Theor. Phys. 53, 77 (1973)

14. Kato, T.: Perturbation theory for linear operators. Berlin, Heidelberg, New York : Springer 1966

15. Krein, M.G., Rutman, M.A.: Usp. Mat. Nauk 3, 3 (1948), Transl. Am. Math. Soc. 26 (1950)

16. Kunz, H., Souillard, B.: Random Schrödinger operators and the theory of disordered systems, some rigorous results, à paraître dans les actes du colloque international "Random fields : rigorous results in statistical mechanics and field theory" juin 1979, Esztergom, Hongrie

17. Matsuda, H., Ishii, K. : Supp. Prog. Theor. Phys. 45, 56 (1970)

18. Molchanov, S.A.: Math. USSR Izvestija, 42(1978), Trad. 12, 69 (1978)

19. Mott, N.: Rev. Mod. Phys. 50, 203 (1978)

20. Mott, N., Twose, W.D.: Adv. Phys. 10, 107 (1961)

21. O'Connor, A.J.: Commun. Math. Phys. 45, 63 (1975)

22. Pastur, L.A.: Russian Math. Surv. 28, 1 (1973)

23. Pastur, L.A.: Spectrum of random Jacobi matrices and Schrödinger equations with random potential in the whole axis (en russe), prétirage, FNINT Akad. Nauk. Ukr. S.S.R., Kar'kov (1974), non publié

24. Reed, M., Simon, B.: Methods of modern mathematıcal physics, Vol. I à IV. New York: Academic Press 1972-1979

25. Royer, G. Croissance exponentielle de produits markoviens de matrices aléatoires, prétirage Université Pierre et Marie Curie. Paris (1979)

26. Ruelle, D.: Nuovo Cimento A 61, 655 (1969)

27. Schwartz, L.: Cours de l'Ecole Polytechnique, Tome I et II. Paris: Hermann 1970

28. Schwartz. L.: Radon measures on arbitrary topological spaces and cylindrical measures. Oxford: Oxford University Press (1973)

29. Stieltjes, T.: Oeuvres complètes.

30. Thouless, D.: Phys. Rep. 13, 93 (1974)

31. Verheggen, T.: Commun. Math. Phys. 68, 69 (1979)

32. Weaire, D., Thorpe, M.F.: Phys. Rev. 4, 2508 (1971)

33. Yoshioka, Y.: Proc. Jpn. Acad. 49, 665 (1973)

Transmis par B. Simon

Reçu le 22 avril 1980 